\title{
Effect of different chemical bonds in pegylation of zinc protoporphyrin that affects drug release, intracellular uptake, and therapeutic effect in the tumor
}

Kenji Tsukigawa $^{\text {a,b,c }}$, Hideaki Nakamura ${ }^{\text {a,c }}$, Jun Fang ${ }^{\text {a,c, } *}$, Masaki Otagiri ${ }^{\text {a,b }}$, Hiroshi Maeda ${ }^{\text {a,* }}$

${ }^{\mathrm{a}}$ Institute for Drug Delivery Science, Sojo University, ${ }^{\mathrm{b}}$ Laboratories of Pharmacokinetics and

${ }^{c}$ Microbiology and Oncology, Faculty of Pharmaceutical Sciences, Sojo University, Ikeda 4-22-1, Nishi-ku, Kumamoto, 860-0082, Japan

*Corresponding Author: Hiroshi Maeda, and Jun Fang.

E-mail address: hirmaeda@ph.sojo-u.ac.jp (HM); fangjun@ph.sojo-u.ac.jp (JF)

Postal address: Institute for Drug Delivery Science, Sojo University, Ikeda 4-22-1, Nishi-ku, Kumamoto, 860-0082, Japan.

Tel.: +81 96326 4114; fax: +810963263185 


\begin{abstract}
Pegylated zinc protoporphyrin (PEG-ZnPP) is a water-soluble inhibitor of heme oxygenase-1. In this study, we prepared two types of PEG-ZnPP conjugates with different chemical bonds between PEG and ZnPP, i.e., ester bonds and ether bonds, where both conjugates also contain amide bonds. Cleavability of these bonds in vitro and in vivo, especially cancer tissue, and upon intracellular uptake, was investigated in parallel with biological activities of the conjugates. Each conjugate showed different cleavability by plasma esterases and tumor proteases, as revealed by HPLC analyses. PEG-ZnPP with ester bond (esPEG-ZnPP) was more sensitive than PEG-ZnPP with ether bond (etPEG-ZnPP) for cleavage of PEG chains. etPEG-ZnPP showed no cleavage of PEG chains and had lower intracellular uptake and antitumor activity than did esPEG-ZnPP. The degradation of esPEG-ZnPP appeared to be facilitated by both serine and cysteine proteases in tumor tissues, whereas it was significantly slower in normal organs except the liver. Depegylated products such as free ZnPP had higher intracellular uptake than did intact PEG-ZnPP. We also studied hydrolytic cleavage by blood plasma of different animal species; mouse plasma showed the fastest cleavage whereas human plasma showed the slowest. These results suggest that ester-linked conjugates manifest more efficient cleavage of PEG, and greater yield of the active principle from the conjugates in tumor tissues than in normal tissues. More efficient intracellular uptake and thus an improved therapeutic effect with ester-linked conjugates are thus anticipated with fain stability, particularly in human blood.
\end{abstract}

Keywords: PEG-ZnPP, PEG-dilemma, PEG-cleavability, tumor proteases, intracellular uptake, antitumor effect 


\section{Introduction}

Polyethylene glycol (PEG) is essentially a nontoxic biocompatible polymer, and widely utilized in conjugation of pharmaceutical proteins and other drugs so as to mask antigenecity of proteins and improve water solubility of the conjugated drugs as well as prolonging blood circulation time [1-3]. The PEG moiety forms an aqueous layer on the protein or drug surface, which also suppresses protein adsorption and recognition by the reticuloendothelial system, thus conferring stealth characteristics [4,5]. Macromolecular drugs (>40 kDa), after modification with such polymers, also show higher accumulation in tumors than do parental low-molecular-weight drugs, due to the enhanced permeability and retention (EPR) effect $[3,6,7]$. However, despite tumor selective delivery of pegylated macromolecular antitumor agents, pegylation hampers the therapeutic effect on tumor cells because of decreased intracellular uptake of the conjugates [8]. This problem is now referred as PEG-dilemma $[8,9]$.

To overcome this drawback, many researchers have investigated the possibility of utilizing cleavage of PEG at tumor sites. For this purpose, several mechanisms of cleaving PEG have been reported using enzyme-cleavable peptide-linker for proteases such as matrix metalloproteinases (MMP) and cathepsin B, and esterases, as well as $\mathrm{pH}$-sensitive linkers such as hydrazone-bonded PEG [9-13].

Another PEG-related problem is the formation of antibodies (IgM) against PEG conjugates [14], which might cause undesirable rapid clearance of pegylated drugs from the blood circulation. Various factors such as the size of pegylated drugs, surface density of PEG, and chain length of PEG in eliciting anti-PEG-IgM were investigated previously $[15,16]$.

Previously, we developed pegylated zinc protoporphyrin (PEG-ZnPP) as a water-soluble macromolecular antitumor agent [17]. ZnPP is almost insoluble in water and it inhibits heme oxygenase-1 (HO-1), which is a key enzyme in heme catabolism [18]. HO-1 is also called heat shock protein 32 (HSP-32), and it is also known as a survival factor of tumor cells. On the contrary to HO-2 which is a constitutive isoform of $\mathrm{HO}, \mathrm{HO}-1$ is an enzyme that is induced by various stimuli such as ultraviolet radiation, oxidative stress, metalloporphyrins, heavy metals, and nitric oxide [18-22]. HO-1 is also reported to exhibit antioxidative and antiapoptotic effects during different cellular stresses. In addition, high HO-1 expression in various solid tumors is well known [18,20,21]. In many cancer cells, antioxidant enzymes such as glutathione peroxidase, catalase, and superoxide dismutase are down-regulated or are 
absent $[18,23]$. Therefore, biological function of HO-1 is thus important for protection of cancer cells against oxidative stress of endogenous reactive oxygen species or that by anticancer agents [21]. It was also reported that $\mathrm{ZnPP}$ exhibited antitumor effect by other mechanisms such as oncogene regulation [24,25].

The poor water solubility of ZnPP limits its therapeutic application, while we can successfully improve its water solubility by pegylation: PEG-ZnPP forms micelles and demonstrated high water solubility [17]. Moreover, PEG-ZnPP accumulated in tumors more preferably according to the EPR effect $[6,7,26,27]$. However, we also found that PEG-ZnPP had lower intracellular uptake than did free ZnPP [28,29], and thus another example of PEG-dilemma in PEG-ZnPP.

In our recent studies, we found that PEG-ZnPP underwent hydrolytic cleavage of PEG chains inside or near cells in vitro [28]. Cells may be able to internalize the depegylated $\mathrm{ZnPP}$ derivatives more rapidly. Therefore, freely liberated depegylated ZnPP derivatives may thus show higher cell uptake and hence better therapeutic activity than PEG-ZnPP. This means facilitated depegylation may be important for its biological activity.

In the present study described here, we aimed to clarify the mechanism and kinetics of degradation of PEG-ZnPP in blood circulation and in tumor tissues by utilizing different types of bonds between PEG and ZnPP. To prepare different bonds, we used two types of PEG, namely, PEG containing ester bond, and ether bond, and we refer here ester-bonded PEG-ZnPP (esPEG-ZnPP) and ether-bonded PEG-ZnPP (etPEG-ZnPP). Also, amide bonds are common bond in both esPEG-ZnPP and etPEG-ZnPP.

At first, we investigated the in vitro PEG cleavability of these two types of PEG-ZnPP in blood and in tumor tissues by using blood plasma and tumor tissue homogenates, respectively. Furthermore, we used cultured tumor cells to study the intracellular uptake and cytotoxicity of esPEG-ZnPP and etPEG-ZnPP in vitro. Then, we investigated antitumor effect, tissue distribution, and the PEG cleavability of two types of PEG-ZnPP in vivo. 


\section{Materials and Methods}

\subsection{Materials}

Protoporphyrin IX (PP) and leupeptin were obtained from Sigma Aldrich Chemical Co. (St. Louis, MO). Free ZnPP was obtained from Frontier Scientific, Inc. (Salt Lake City, UT). $N$-Succinimidyl PEG containing ester bond (ME-20CS) with a mean molecular weight of 2322 and $N$-succinimidyl PEG containing ether bond (ME-20AS) with a mean molecular weight of 2280 were obtained from NOF (Tokyo, Japan). Fetal bovine serum was obtained from GIBCO (Grand Island, NY). 3-(4,5-Dimethyl-2-thiazolyl)-2,5-diphenyl-2H-tetrazolium bromide (MTT) was purchased from Dojindo Chemical Laboratories (Kumamoto, Japan). Succinic anhydride, RPMI 1640 medium, Dulbecco's Modified Eagle Medium (DMEM), penicillin G, streptomycin, diisopropylfluorophosphate (DFP), phenylmethylsulfonylfluoride (PMSF), N-tosyl-L-phenylalaninechloromethyl ketone (TPCK), iodoacetamide, and $\mathrm{N}$-acetylcysteine were purchased from Wako Pure Chemical (Osaka, Japan). All other reagents were reagent grade and used without further purification. Samples of human colorectal tumor tissues (well-differentiated adenocarcinoma) as well as the neighboring normal colon tissues were supplied from National Hospital Organization Kumamoto Medical Center (Kumamoto, Japan). Use of the human tumor tissues was approved by the ethical committee of National Hospital Organization Kumamoto Medical Center.

\subsection{Synthesis of two types of PEG-ZnPP}

Two different types of PEG were conjugated to PP as described previously with some modification [17]. Briefly, before pegylation, PP (100 mg in $20 \mathrm{~mL}$ of tetrahydrofuran) was first conjugated with ethylenediamine $(2.4 \mathrm{~mL})$ via formation of an amide bond. The crude bis-diaminoethyl protoporphyrin (PPED) obtained was adsorbed to activated alumina and was washed five times with chloroform containing 0.2\% ethylenediamine. PPED was then eluted from activated alumina with chloroform containing 5\% ethylenediamine. To prepare esPEG-ZnPP (Figure 1A), PEG containing ester bond (ME-20CS, NOF) (54 mg) was reacted with the PPED (5 mg in $20 \mathrm{~mL}$ of chloroform) at room temperature for $4 \mathrm{~h}$. The reaction mixture was applied to an activated alumina column $(25 \times 50 \mathrm{~mm})$ equilibrated and eluted with chloroform to remove unreacted PPED. A 100 molar excess of zinc acetate powder was then added to the conjugate in chloroform with stirring at room temperature for $2 \mathrm{~h}$, thereby 
chelating the zinc. Chloroform was removed by evaporation in vacuo at $40{ }^{\circ} \mathrm{C}$, which yielded esPEG-ZnPP as a dark reddish powder. esPEG-ZnPP was further purified by using the Labscale Tangential Flow Filtration System (Millipore, Bedford, MA) with a 10-kDa cutoff membrane filter under pressure, followed by lyophilization. The $\mathrm{ZnPP}$ content was $10 \%$ $(w t / w t)$.

etPEG-ZnPP (Figure 1B) was prepared by using a procedure similar to that for esPEG-ZnPP except with using PEG containing ether bond (ME-20AS, NOF) for pegylation. Both esPEG-ZnPP and etPEG-ZnPP contain two amides derived from ethylenediamine (Figure 1A, B).

2.3. Synthesis of bis-diaminoethyl zinc protoporphyrin (ZnPPED) and succinyl ZnPPEDs (mono- and bis-succinyl ZnPPED)

ZnPPED (Figure 1D) was synthesized as a putative degradation product of PEG-ZnPP after depegylation by hydrolysis of the amide bonds adjacent to the PEG chains. PPED was dissolved in chloroform after which 10 molar excess zinc acetate was added with stirring at room temperature for $2 \mathrm{~h}$ so as to chelate zinc. After the reaction, chloroform was removed by evaporation. ZnPPED thus obtained was washed with distilled water five times, followed by lyophilization, yielding ZnPPED powder.

In addition to free ZnPP (Figure 1C) and ZnPPED (Figure 1D), succinyl ZnPPEDs (mono- and bis-succinyl ZnPPED) (Figure 1E, F) were candidate products for depegylation. Succinyl ZnPPEDs were synthesized by using a 10 molar excess of succinic anhydride in tetrahydrofuran under stirring at room temperature for $5 \mathrm{~h}$ reacting with ZnPPED.

\subsection{High performance liquid chromatography (HPLC)}

HPLC was performed to separate cleavage products of PEG-ZnPP by using LC-2000Plus series HPLC system (JASCO, Tokyo, Japan) equipped with PU-2080 pump, UV-2075 UV/Visible detector (Integrator output scale was 1 V/1.0 AU), and 807-IT integrator (The settings were: ATTENUATION: $32 \mathrm{mV}$ F. S.; CHART SPEED: $5 \mathrm{~mm} / \mathrm{min}$; MIN WIDTH: $0.10 \mathrm{~min}$; MIN HEIGHT: $100 \mu \mathrm{V}$; MIN AREA: $100 \mu \mathrm{V}$; DRIFT; $0 \mu \mathrm{V} / \mathrm{min}$; DOUBLE WIDTH TIME; 0 min; NOISE LEVEL: $10 \mu \mathrm{V}$ ). The column was Asahipak GF-310 HQ column $(7.5 \times 300 \mathrm{~mm})$ (Showa Denko, Tokyo, Japan), and the prefilter was Asahipak GF-1G 7B column $(7.5 \times 50 \mathrm{~mm})$ (Showa Denko, Tokyo, Japan). The mobile phase 
consisted of $70 \%$ methanol, 30\% dimethyl sulfoxide (DMSO), and $0.001 \%$ trifluoroacetic acid at a flow rate of $0.8 \mathrm{~mL} / \mathrm{min}$; eluate was monitored at $415 \mathrm{~nm}$ for $\mathrm{ZnPP}$. The injection volume was $20 \mu \mathrm{L}$. PEG-ZnPP and its degradation products were quantified by their peak areas, and in most studies they were described as percent ratio to the total peak areas.

\subsection{MALDI-TOF MS}

The molecular weights of PEG-ZnPP and cleavage products of PEG-ZnPP were analyzed by using MALDI-TOF MS (Autoflex II TOF/TOF, Bruker Daltonics, Bremen, Germany).

For analysis of PEG-ZnPP, $10 \mu \mathrm{L}$ of PEG-ZnPP $(100 \mu \mathrm{g} / \mathrm{mL})$ in THF was mixed with $10 \mu \mathrm{L}$ of dithranol (10 mg/mL, Bruker Daltonics, Bremen, Germany) dissolved in THF. Then, $1 \mu \mathrm{L}$ of the mixture was spotted on a target plate (MTP 384 target plate ground steel T F, Bruker Daltonics, Bremen, Germany) and allowed to dry at room temperature. Mass spectra were measured with flexControl (Bruker Daltonics, Bremen, Germany). The settings were: method: reflector positive mode; shots: 200; frequency: 100; laser power: 10-30\%. And the mass spectra were analyzed with flexAnalysis (Bruker Daltonics, Bremen, Germany).

The cleavage products of PEG-ZnPP were isolated by HPLC respectively as described above. About $5 \mathrm{~mL}$ of each peak fraction was collected and methanol in mobile phase was removed by evaporation in vacuo at $40{ }^{\circ} \mathrm{C}$. And $10 \mathrm{~mL}$ of distilled water was added, and lyophilization. The obtained powder was dissolved in $20 \mu \mathrm{L}$ of THF and mixed with $20 \mu \mathrm{L}$ of dithranol $(10 \mathrm{mg} / \mathrm{mL})$ dissolved in THF. Then, Then, $1 \mu \mathrm{L}$ of the mixture was spotted on a target plate (MTP 384 target plate ground steel T F) and allowed to dry at room temperature. Mass spectra were measured with flexControl. The settings were: method: reflector positive mode; shots: 200; frequency: 100; laser power: 10-30\%. And the mass spectra were analyzed with flexAnalysis.

\subsection{Stability/PEG cleavability of PEG-ZnPPs in vitro: Treatment of PEG-ZnPPs with blood} plasma, tumor homogenates, and normal tissue homogenates

To investigate the stability of two PEG-ZnPPs in blood, es- and et- PEG-ZnPP (1 $\mathrm{mg} / \mathrm{mL}$ ) was incubated at $37{ }^{\circ} \mathrm{C}$ in $\mathrm{PBS}(0.01 \mathrm{M}$ phosphate-buffered $0.15 \mathrm{M}$ saline, $\mathrm{pH} 7.4)$ containing $10 \%$ blood plasma of different animal species (human, rabbit, mouse, or rat). In some experiments, esPEG-ZnPP was further incubated in the presence of a serine-type 
esterase/protease inhibitor, DFP (1 mM) which was diluted by PBS (pH 7.4). At the indicated times, $10 \mu \mathrm{L}$ of each incubation mixture was taken to add to $1 \mathrm{~mL}$ of the mobile phase of the HPLC. Then it was centrifuged to remove insoluble materials, and the supernatant was subjected to HPLC.

To investigate the PEG cleavability of two PEG-ZnPPs in tumor tissue, es- and etPEG-ZnPP (1 mg/mL) was incubated at $37{ }^{\circ} \mathrm{C}$ in $50 \mathrm{mM}$ phosphate buffer (pH 6.2) containing supernatant of mouse sarcoma 180 (S-180) or human colorectal tumor (well-differentiated adenocarcinoma) homogenates (final conc.: 2 or $5 \mathrm{mg} / \mathrm{mL}$ protein). With supernatant of S-180 tumor homogenates at $5 \mathrm{mg} / \mathrm{mL}$ protein, esPEG-ZnPP was incubated in the absence or presence of protease inhibitors, TPCK (1 mM), PMSF (1 mM), leupeptin (10 $\mu \mathrm{g} / \mathrm{mL})$, and iodoacetamide (1 mM). TPCK and PMSF were dissolved in ethanol and DMSO respectively, and final concentration of ethanol and DMSO in incubation mixture was $0.1 \%$ (v/v). Leupeptin and iodoacetamide were dissolved in $50 \mathrm{mM}$ phosphate buffer $(\mathrm{pH}$ 6.2). With supernatant of human colorectal tumor at $5 \mathrm{mg} / \mathrm{mL}$ protein, esPEG-ZnPP was incubated in the absence or presence of $N$-acetylcysteine $(100 \mu \mathrm{M}$ or $1 \mathrm{mM}) . N$-acetylcysteine was dissolved in $50 \mathrm{mM}$ phosphate buffer ( $\mathrm{pH}$ 6.2). At the indicated times, HPLC analysis was performed as described above.

To investigate the PEG cleavability of esPEG-ZnPP in normal tissues, esPEG-ZnPP (1 $\mathrm{mg} / \mathrm{mL}$ ) was incubated at $37^{\circ} \mathrm{C}$ in PBS (pH 7.4) containing supernatant of mouse normal tissues (spleen, kidney, liver) homogenates (final conc.: $2 \mathrm{mg} / \mathrm{mL}$ protein) or human normal colon tissue homogenates (final conc.: $5 \mathrm{mg} / \mathrm{mL}$ protein). At the indicated times, HPLC analysis was performed as described above.

\subsection{Cell culture}

Human chronic myelogenous leukemia cells (K562) were cultured as usual in RPMI 1640 medium with inactivated $10 \%$ fetal bovine serum, $100 \mathrm{U} / \mathrm{mL}$ penicillin $\mathrm{G}$, and 100 $\mu \mathrm{g} / \mathrm{mL}$ streptomycin at $37{ }^{\circ} \mathrm{C}$ in $5 \% \mathrm{CO}_{2} / 95 \%$ air.

Human esophageal squamous carcinoma cells (KYSE150), supplied by Dr. Hiroshi Kumimoto, Aichi Cancer Center Research Institute, and human colon cancer cells (HT-29) were cultured similarly to K562 cells but in DMEM.

\subsection{Quantification of ZnPP derivatives in cells}


K562 cells $\left(5 \times 10^{5}\right.$ cells/well $)$ were placed in a 12 -well plate $(22.1 \mathrm{~mm}$ in well diameter). After overnight incubation, cells were treated with esPEG-ZnPP, etPEG-ZnPP, free ZnPP, or ZnPPED at $20 \mu \mathrm{M}$ each (ZnPP equivalent) for the indicated times. The esPEG-ZnPP and etPEG-ZnPP were dissolved in PBS (pH 7.4); free ZnPP and ZnPPED were dissolved in DMSO. After incubation for indicated times, cells were collected by centrifugation and washed three times with PBS at room temperature. Amounts of the intracellular ZnPP derivatives were quantified by fluorescence intensity by fluorescence-activated cell sorter (FACS) analysis using FACSCalibur (Nippon Becton Dickinson Co., Ltd., Tokyo, Japan). For analysis of the desired cell peaks, $1 \times 10^{4}$ cells were collected, and the mean fluorescence intensity of each sample was recorded and analyzed.

Intracellular uptake of ZnPP derivatives was also analyzed using HT-29 cells. HT-29 cells $\left(5 \times 10^{5}\right.$ cells/well $)$ were placed in a 12-well plate. After overnight incubation, cells were treated with $\mathrm{ZnPP}$ derivatives at $20 \mu \mathrm{M}$ each (ZnPP equivalent) for the indicated times. After incubation for the indicated times, cells were washed with PBS twice, trypsinized, and were resuspended in methanol. Then, cells were sonicated $(30 \mathrm{~W}, 30 \mathrm{~s}$, Hielscher, Teltow, Germany) at ice chilled condition, followed by centrifugation to collect supernatant containing ZnPP derivatives, and fluorescence intensity (excitation at $420 \mathrm{~nm}$, emission at $590 \mathrm{~nm}$ ) of the methanol extract was measured using fluorescence spectrophotometer (FP-6600; JASCO, Tokyo, Japan).

\subsection{Confocal fluorescence microscopic analysis of $\mathrm{ZnPP}$ derivatives in cells for intracellular} uptake

To visualize ZnPP derivatives in the cells, confocal microscopy was performed with Nikon Eclipse TE2000-E Confocal Microscope (Nikon, Tokyo, Japan). KYSE150 cells were cultured in DMEM without phenol red with a round glass bottom petri dish $(\varphi=35 \mathrm{~mm}$, Matsunami Glass, Osaka, Japan). Cells were treated with at $30 \mu \mathrm{M}$ each of esPEG-ZnPP, etPEG-ZnPP, free ZnPP, or ZnPPED for the indicated times at $37{ }^{\circ} \mathrm{C}$. After incubation, analysis of ZnPP derivatives in cells carried out at $488 \mathrm{~nm}$, and fluorescence was detected at $565-615 \mathrm{~nm}$.

\subsection{Cytotoxicity assay}

In vitro cytotoxicity of ZnPP derivatives was investigated by using the MTT assay. 
K562 cells and HT-29 cells were plated in 96-well culture plates (3000 cells/well). After overnight incubation, esPEG-ZnPP, etPEG-ZnPP, free ZnPP, or ZnPPED was added. The MTT assay was performed to quantify the viable cells with absorbance at $570 \mathrm{~nm}$ after $72 \mathrm{~h}$ of culture, by a microplate reader (Infinite M200 PRO; Tecan Japan Co., Ltd., Kanagawa, Japan).

\subsection{Antitumor effect of es- and et-PEG-ZnPP in vivo}

Routine care and maintenance of animals were carried out in accordance with the institutional guidelines of the Institutional Animal Care and Use Committee of Sojo University. In vivo antitumor activity of two types of PEG-ZnPP was examined with S-180 tumor bearing male ddY mice. Male ddY mice, 6 weeks of age, were purchased from Kyudo Co., Ltd (Saga, Japan). S-180 cells $\left(2 \times 10^{6}\right.$ cells $)$ were implanted s.c. in the dorsal skin of ddY mice. When tumor reached to diameter of about 3-5 mm, $15 \mathrm{mg} / \mathrm{kg}$ of $\mathrm{ZnPP}$ equivalent drugs in saline was administered intravenously (i.v.) via the tail vein. Tumor volume $\left(\mathrm{mm}^{3}\right)$ was calculated as $\left(\mathrm{W}^{2} \times \mathrm{L}\right) / 2$ by measuring the length $(\mathrm{L})$ and width $(\mathrm{W})$ of the tumor on the dorsal skin.

\subsection{Tissue distribution and cleavability of es- and et-PEG-ZnPP in vivo}

Tissue distribution and the PEG cleavability of es- and et- PEG-ZnPP in vivo were examined by using mouse S-180 tumor bearing male ddY mice. S-180 cells were similarly used as above. When diameter of tumor reached to about $10 \mathrm{~mm}, 40 \mathrm{mg} / \mathrm{kg}$ of $\mathrm{ZnPP}$ equivalent drugs in saline was injected i.v. via the tail vein. At the indicated time after i.v. injection, mice were killed and perfused with physiological saline to remove residual blood, and organs were dissected. The mobile phase of HPLC (500 $\mu \mathrm{L}$ per $100 \mathrm{mg}$ of tissue) was added to the tissue samples, then the samples were homogenized at ice chilled condition and centrifuged to remove insoluble tissue debris. Then, the supernatant was subjected to HPLC as described above. 


\section{Results and Discussion}

3.1. Analyses of the cleavage products of PEG-ZnPPs after treatment with blood plasma or supernatant of tumor tissue homogenates

Figure 2A-i showed the chromatogram of PEG-ZnPP which showed had a main single peak at $7.5 \mathrm{~min}$. When it was treated with $10 \%$ blood plasma or with supernatant of tumor homogenates (final conc.: 2 or $5 \mathrm{mg} / \mathrm{mL}$ protein), several peaks were detected, where peak retention time varied from 7.5 min (intact PEG-ZnPP, Figure 2A-i) to 8.3, 11.2, 11.8, and 12.8 min (cleavage products) as shown in Figure 2A-ii (a sample of esPEG-ZnPP treated with 10\% mouse blood plasma for $6 \mathrm{~h}$ ). The precision, repeatability of this HPLC method was tested by using PEG-ZnPP incubated in supernatant of S-180 tumor homogenates (final conc.: $5 \mathrm{mg} / \mathrm{mL}$ protein) for $72 \mathrm{~h}$; the relative standard deviations (\%RSD) were less than $0.1 \%$ for retention time of each peak (Table S1) and less than $3.7 \%$ for peak area of each peak (Table S2). Total of peak area of all peaks showed no significant change between $0 \mathrm{~h}$ and $72 \mathrm{~h}$ after the incubation (Table S3). These results may at least partly support the repeatability and reliability of this HPLC assay. In addition, the lower limit of detection for PEG-ZnPP in this analysis was $1 \mathrm{ng}$ or $0.1 \mathrm{ng}$ of $\mathrm{ZnPP}$.

The intact PEG-ZnPP (peak 1) has a mean molecular weight of about 5000, which was also supported by MALDI-TOF MS (Figure S2A). The peak 2 had a mean molecular weight of about 3000 (Figure S2B), and it was detected only with esPEG-ZnPP (see Figure 3B, D). From these results, peak 2 was considered as mono esPEG-ZnPP, with one PEG chain detached at the ester bond (see Figure S1). Though MALDI-TOF MS could not accurately determine the molecular weight of the peaks 3-5, products with molecular weight of more than 1000 were not detected (Figure S2C-E), suggesting they are further depeglated products. When we compared these peaks with free ZnPP (12.8 min), ZnPPED (6.5 min), and succinyl ZnPPEDs (11.2 and 11.8 min) (Figure 2B), we found that peak 5 was agreement with the peak of free ZnPP; peaks 3 and 4 were accorded with the peaks of succinyl ZnPPEDs. These findings support that peaks 3-5 may be completely depegylated products. In addition, there was no peak corresponding to ZnPPED which was also completely depegylated product in the cleaved products as seen by HPLC (Figure 2A-ii, B). 
Pegylated antitumor drugs must be stable and retain their macromolecular integrity during blood circulation. As a result, they can achieve high accumulation in tumor due to the EPR effect $[6,30]$. In this study, we investigated the stability of both es- and et- PEG-ZnPP under incubation with $10 \%$ blood plasma from various animal species. Because peak 1 and 2 , which were pegylated $\mathrm{ZnPP}$ derivatives, were important for macromolecular integrity/micelle formation during blood circulation, changes of peak 1 and 2 were used to evaluate stability of es- and et- PEG-ZnPP in circulation. Figure 3A shows the comparison of peak 1 (intact PEG-ZnPP) degradation of esPEG-ZnPP and etPEG-ZnPP. Peak 1 of etPEG-ZnPP showed no apparent change for $48 \mathrm{~h}$ and etPEG-ZnPP remained uncleaved, whereas esPEG-ZnPP was degraded in a time-dependent manner, with the decrease being faster in mouse and rat plasma than in human and rabbit plasma. Similar results were noted for peak 2 (Figure 3B): at $24 \mathrm{~h}$ after incubation, the degradation of peak 2 in human plasma was about 1/8 of that of mouse and rat plasma.

In our previous study, we found that both esPEG-ZnPP and etPEG-ZnPP had a longer blood circulation time, resulting in higher accumulation in tumors because of the EPR effect, than that seen for free $\mathrm{ZnPP}$ in a mouse model [26,27]. These facts together with our present findings indicated that although esPEG-ZnPP was relatively cleavable in blood circulation than etPEG-ZnPP as described above, the pharmacokinetic properties of this PEG-ZnPP conjugate were sufficient for achieving EPR effect, e.g., more than 60-80\% of esPEG-ZnPP

remain pegylation (peak 1 and 2) after 4-6 h incubation in blood plasma (Figure 3). A more important and interesting finding is the significant species differences in cleavability of the PEG: degradation of peaks 1 and 2 in human plasma was much slower than that in mouse and rat plasma. These results suggested that esPEG-ZnPP was more stable in human plasma than in other animal plasma, and thus would exhibit a superior accumulation in tumors in patients in view of the EPR effect.

\subsection{Effect of DFP on stability of esPEG-ZnPP}

From the data in Figure $3 \mathrm{~A}$ and $\mathrm{B}$, we have observed a significant difference in stability of esPEG-ZnPP in plasma from different animal species. We then investigated the causes of cleavage of PEG from esPEG-ZnPP and species differences in plasma. Species differences in activities of hydrolytic enzymes such as esterase in blood plasma have been reported [31]. We thus examined the stability of esPEG-ZnPP in $10 \%$ human or mouse plasma 
in the presence of DFP $(1 \mathrm{mM})$, a serine-type esterase/protease inhibitor. Figure $3 \mathrm{C}$ shows that DFP suppressed the peak 1 degradation in mouse plasma but not much in human plasma. The results observed for changes in peak 2 was more notable for mouse plasma but not much in human plasma (Figure 3D). DFP had less effect in human plasma compared with that in mouse plasma. These findings suggest that esterases such as carboxylesterase, which is abundant in mouse blood plasma [31], are involved in depegylation of esPEG-ZnPP in blood plasma and may lead to the species differences in stability. In addition, because DFP is also a serine protease inhibitor, these findings suggest that serine proteases may also involve in cleavage between PEG and ZnPP in plasma, which warrants additional investigation.

\subsection{Cleavability of the PEG from es- and et-PEG-ZnPP after incubation with supernatant of mouse tumor homogenates}

Stability of the pegylated drugs during blood circulation is a prerequisite for their delivery to tumor sites or lesions, after which depegylation is needed for efficient intracellular uptake and therapeutic effect. Namely, it is important that completely depegylated products are cleaved from PEG-ZnPP in tumor tissue. Therefore, yield of peak 3, 4 and 5 which were presumed to be completely depegylated products, were then evaluated.

To investigate the depegylation from esPEG-ZnPP and etPEG-ZnPP at the tumor site, we incubated esPEG-ZnPP and etPEG-ZnPP in $50 \mathrm{mM}$ phosphate buffer (pH 6.2) with supernatant of mouse S-180 tumor homogenates (final conc.: 2 or $5 \mathrm{mg} / \mathrm{mL}$ protein). After incubation of esPEG-ZnPP without supernatant of tumor homogenates, peaks 3 and 4 were not generated up to $72 \mathrm{~h}$ (Figure 4A, B), and only about $5 \%$ of peak 5 was generated after 72 $\mathrm{h}$ incubation (Figure 4C). In contrast, the cleavage of esPEG-ZnPP increased after incubation with supernatant of tumor homogenates in a dose- and time-dependent manner (Figure 4A, B, C). However, no generation of these products occurred in etPEG-ZnPP after incubation with supernatant of tumor homogenates (final conc.: $5 \mathrm{mg} / \mathrm{mL}$ protein) (Figure 4A, B, C). These results suggest that PEG was not removed from etPEG-ZnPP by the supernatant of tumor homogenates.

\subsection{Effect of protease inhibitors on cleavability of the PEG from esPEG-ZnPP}

To investigate the factors involved in cleavage of the PEG from esPEG-ZnPP at a tumor site, we focused on serine and cysteine proteases, which are highly expressed in tumors 
and facilitate tumor invasion and metastasis [32]. We investigated the effect of protease inhibitors on cleavability of the PEG during incubation with supernatant of mouse S-180 tumor homogenates (final conc.: $5 \mathrm{mg} / \mathrm{mL}$ protein). We used TPCK (1 mM), PMSF (1 mM), leupeptin $(10 \mu \mathrm{g} / \mathrm{mL})$, and iodoacetamide $(1 \mathrm{mM})$ in this study. As Figure 5A illustrates, PMSF almost completely suppressed the generation of peak 3 through $72 \mathrm{~h}$. Other inhibitors also suppressed the generation of peak 3 , and the inhibitory effect was found in the order of TPCK > iodoacetamide > leupeptin, suggesting both serine and cysteine proteases appear to be involved in the depegylation from esPEG-ZnPP at the tumor tissue. Similar results were observed for peak 4, but the protease inhibitors in general had a reduced effect (Figure 5B). However, all protease inhibitors promoted the generation of peak 5 at $72 \mathrm{~h}$ (Figure 5C), which suggests that other proteases may also participate in the cleavage of PEG-ZnPP. Serine and cysteine proteases, which were involved in the generation of peaks 3 and 4 , may be suppressed by TPCK, PMSF, leupeptin, or iodoacetamide, which may result in the accumulation of peak 5 .

We also investigated the cleavability of the PEG from esPEG-ZnPP by using homogenates of other tissues from male ddY mouse: the spleen, the kidney, and the liver. Figure 6 shows the generation of cleavage products, as a total of peaks 3-5 combined, after incubation with supernatants of homogenates of these normal tissues at $\mathrm{pH} 7.4$ and with supernatant of tumor homogenates at $\mathrm{pH} 6.2$ (final conc.: $2 \mathrm{mg} / \mathrm{mL}$ protein). Supernatants of normal tissues also generated cleavage products, with the yield being in the order of liver > tumor > kidney and spleen. This result may reflect high hydrolytic enzymes in the liver. This indicates if esPEG-ZnPP is delivered to tumor it would generate more depegylated $\mathrm{ZnPP}$ derivatives than other normal tissue, except liver. This is preferable point. $\mathrm{ZnPP}$ is not toxic to the liver [26], thus results are favorable for more tumor selective toxicity.

\subsection{Cleavability of the PEG from esPEG-ZnPP after incubation with supernatant of human} tumor homogenates

We investigated similar cleavability of PEG chains from esPEG-ZnPP by using homogenates of human colorectal tumor (well-differentiated adenocarcinoma), as well as mouse S-180 tumor. As shown in Figure 7A, cleavage products (total of peaks 3-5 combined) were generated after incubation with supernatant of human colorectal tumor homogenates (final conc.: $5 \mathrm{mg} / \mathrm{mL}$ protein) at $\mathrm{pH} 6.2$. 
From the data in Figure 5, we found that the depegylation of esPEG-ZnPP was facilitated by both serine and cysteine proteases in tumor tissues. Cysteine proteases are activated by more reducing condition and agents such as 2-mercaptoethanol and dithiothreitol by preserving active thiol residue. For this purpose, $N$-acetylcysteine was used. $\mathrm{N}$-acetylcysteine did facilitated generation of cleaved products (total of peaks 3-5 combined) (Figure 7B). This result suggests that esPEG-ZnPP may be more effectively cleaved in vivo setting of tumor, where proteases such as cysteine proteases, e.g. cathepsin B, were known up-regulated [32,33].

Moreover, we compared cleavability of PEG from esPEG-ZnPP after incubation with supernatants of human colorectal tumor at $\mathrm{pH}$ 6.4, and that of normal large intestine at $\mathrm{pH} 7.4$. After $24 \mathrm{~h}$ of incubation, yield of cleavage products (total of peaks 3-5 combined) after incubation with supernatant of homogenate of human normal large intestine was lower than that with supernatant of corresponding colorectal tumor (Figure 7C). This result indicates that more preferential cleavage of PEG may proceed in tumor tissues than in normal tissues.

\subsection{Intracellular uptake and cytotoxicity of PEG-ZnPPs and the cleavage products in vitro,} and antitumor effect of PEG-ZnPPs in vivo

Intracellular uptake of pegylated drugs was reported that it was improved after depegylation [8]. We examined this by using K562 cells and HT-29 cells whether depegylated products would show higher intracellular uptake than intact PEG-ZnPPs. For this study, free ZnPP (Figure 1C) and ZnPPED (Figure 1D) were used as references. Figure 8A shows that the depegylated products were incorporated into cells much faster than esPEG-ZnPP and etPEG-ZnPP in K562 cells. Similar results were observed in HT-29 cells (Figure 8B). Confocal microscopic analysis with KYSE150 cells after $2.5 \mathrm{~h}$ of incubation confirmed these findings, as seen in Figure 8C. More important finding, esPEG-ZnPP and etPEG-ZnPP were taken up by cells in a time-dependent manner, and esPEG-ZnPP at $8 \mathrm{~h}$ in K562 cells, at $16 \mathrm{~h}$ and $24 \mathrm{~h}$ in HT-29 cells after incubation showed a significantly higher intracellular uptake compared with etPEG-ZnPP (Figure 8A, B).

These findings strongly support the concept of PEG-dilemma, i.e., pegylated drugs generally show slower intracellular uptake than parental non-pegylated drugs. More important, the high uptake of the depegylated products (free ZnPP and ZnPPED) and higher uptake of esPEG-ZnPP than that of etPEG-ZnPP suggested clear advantage of esPEG-ZnPP over 
etPEG-ZnPP, because of its high cleavability in vivo at the tumor site.

To elaborate further, we performed an in vitro MTT assay with K562 cells to compare the cytotoxicity of esPEG-ZnPP, etPEG-ZnPP, free ZnPP, and ZnPPED (Figure 9A, Table 1). As expected, free $\mathrm{ZnPP}\left(\mathrm{IC}_{50}\right.$ of $\left.0.97 \mu \mathrm{M}\right)$ and $\mathrm{ZnPPED}\left(\mathrm{IC}_{50}\right.$ of $\left.0.85 \mu \mathrm{M}\right)$ demonstrated much higher cytotoxicity than esPEG-ZnPP and etPEG-ZnPP. And esPEG-ZnPP showed relatively potent cytotoxicity compared with etPEG-ZnPP ( IC $_{50} 2.88 \mu \mathrm{M}$ vs $39.1 \mu \mathrm{M}$, respectively). Similar results were observed in HT-29 cells (Figure 9B, Table 2). $\mathrm{IC}_{50}$ of free ZnPP, ZnPPED, esPEG-ZnPP, and etPEG-ZnPP were $4.16 \mu \mathrm{M}, 2.82 \mu \mathrm{M}, 27.7 \mu \mathrm{M}$, and >100 $\mu \mathrm{M}$, respectively. These findings explain the difference between the two PEG-ZnPPs in which cleavability reflects more responsible for biological activity. Utilization of PEG via ester linkages may thus be beneficial for development of various PEG-conjugates, and it is not as labile as envisaged. Further, a great difference between different origin of blood plasma was observed (see Figure 3).

Then, in vivo antitumor effect of es- and et- PEG-ZnPP against S-180 tumor was investigated. As shown in Figure 9C, esPEG-ZnPP exhibited significant higher antitumor effect than etPEG-ZnPP, which is accorded with in vitro higher cleavability, intracellular uptake, and cytotoxicity of esPEG-ZnPP than etPEG-ZnPP.

\subsection{Tissue distribution and PEG cleavability of es- and et-PEG-ZnPP in vivo}

Figure 10A shows tissue distribution (described as total of peaks 1-5) of es- and etPEG-ZnPP at $24 \mathrm{~h}$ after i.v. injection in S-180 tumor bearing male ddY mice. At $24 \mathrm{~h}$ after i.v. injection, both types of PEG-ZnPP showed almost similar tumor accumulation. However, most importantly, significant difference was observed in depegylation. Figure 10B shows the amount of each peak of esPEG-ZnPP in each tissue at $24 \mathrm{~h}$ after i.v. injection, where depegylated products (total of peaks 3-5, black bar) were observed in tumor. Meanwhile, etPEG-ZnPP showed only peak 1 (intact etPEG-ZnPP, white bar) largely in each tissue at $24 \mathrm{~h}$ (Figure 10C). For esPEG-ZnPP, the depegylated products (total of peaks 3-5 combined) in the liver was highest, due to unique affinity of protoporphyrin, followed by that in tumor (Figure 10B). Despite of high liver accumulation, no toxicity of esPEG-ZnPP for liver function was found in our previous study [26].

These findings suggest that esPEG-ZnPP will accumulate in tumor tissue by EPR 
effect, and it will remain there for long time, e.g., several days [6], during which time the PEG chains will undergo hydrolytic removal by various proteases in the tumor tissue. The liberated active components will then be internalized quickly by tumor cells as demonstrated in Figure 8A, B, and $\mathrm{C}$, and thus antitumor effect will become apparent. This finding may explain the higher in vivo antitumor activity of esPEG-ZnPP than that of etPEG-ZnPP (Figure 9C).

\subsection{Mechanism of cleavage of PEG from esPEG-ZnPP}

Figure S1 summarizes the conceptual mechanism of cleavage of PEG from esPEG-ZnPP. In this study, we also found that peaks 3-5 were generated together with a decrease in peak 2, which suggested that peaks 3-5 were generated via peak 2 (mono PEG-ZnPP), and not directly from peak 1 (intact PEG-ZnPP). Generation of peak 2 (mono PEG-ZnPP) is probably critical for further degradation. Additional degradation of mono PEG-ZnPP in the tumor tissue may proceed involving various proteases. Amide bonds exist in both es- and et- PEG-ZnPP (Figure 1A, B), appeared not readily cleavable by hydrolytic enzymes, perhaps because of steric hindrance. The putative structures such as the succinyl ZnPPEDs, ZnPPED, and free ZnPP shown in Figure S1 may correspond to the products indicated by peaks 3-5.

Depegylated products of esPEG-ZnPP will be generated by cleavage of ester bonds and amide bonds between PEG and ZnPP. Although amide bond is more stable than ester bond generally, it is also known that amide-linkage in amide prodrugs of amantadine is hydrolyzed by rat liver homogenate [34]. Once PEG is detached, amide bonds derived from ethylenediamine may be gradually cleaved.

Different numbers of methylene group adjacent to ester carbonyl was recently reported to have an impact on the cleavability of ester bonds in vitro [35]. Therefore, use of different carboxylic ester spacers in PEG-ZnPP may control depegylation rate of the PEG conjugate during blood circulation and in the tumor tissue.

\section{Conclusions}

We described herein depegylation of PEG-conjugated ZnPP in view of chemical bond between PEG and the prosthetic group. PEG was cleaved effectively from the bonds in the following order: ester > ether/amide. For the proteolytic cleavage activity in vivo, cysteine proteases and serine proteases (esterases) in solid tumor tissues play more active roles than 
those of normal organs except the liver. This finding indicates more preferential cleavage of the conjugate-linker to PEG in tumor tissues than in normal tissues would occur. Moreover, the cleavage products showed higher intracellular uptake than the original PEG-ZnPP conjugates, which also explains higher in vitro cytotoxicity and in vivo antitumor effect of esPEG-ZnPP compared with non-cleavable etPEG-ZnPP. Thus, esPEG-ZnPP has beneficial facts favoring more tumor selectivity than normal tissues. Namely, the EPR effect driven tumor accumulation, tumor proteases dependent yield of active ZnPP moieties, and upregulated uptake of active $\mathrm{ZnPP}$ moieties into the tumor cells. The present study thus suggests a possible method to overcome PEG-dilemma, by using ester linkage between PEG and low-molecular-weight drugs for the antitumor drug development.

\section{Acknowledgments}

Support from the Ministry of Health, Labour and Welfare (MHLW), Japan for Cancer Specialty Grant (2011-2014) and from the Matching Fund Subsidy for Private Universities from the Ministry of Education, Culture, Sports, Science and Technology (MECSST), Japan, and Adaptable and Seamless Technology Transfer Program (A-STEP) through target-driven R\&D from Japan Science and Technology Agency (JST), for H. Maeda are gratefully appreciated. We are also indebted to Dr. Hiroshi Kumimoto of the Aichi Cancer Center Research Institute (Nagoya, Japan) for the supply of KYSE150 cells, to National Hospital Organization Kumamoto Medical Center for the supply of human tumor tissues, and to Ms. Judith Gandy for the English editing of the manuscript. 


\section{References}

[1] C. Elvira, A. Gallardo, J.S. Roman, A. Cifuentes, Covalent polymer-drug conjugates, Molecules. 10 (2005) 114-125.

[2] A.S. Hoffman, The origins and evolution of "controlled" drug delivery systems, J. Control. Release. 132 (2008) 153-163.

[3] H. Maeda, G.Y. Bharate, J. Daruwalla, Polymeric drugs for efficient tumor-targeted drug delivery based on EPR-effect, Eur. J. Pharm. Biopharm. 71 (2009) 409-419.

[4] A.L. Klibanov, K. Maruyama, V.P. Torchilin, L. Huang, Amphipathic polyethyleneglycols effectively prolong the circulation time of liposomes, FEBS Lett. 268 (1990) 235-237.

[5] M.L. Immordino, F. Dosio, L. Cattel, Stealth liposomes: review of the basic science, rationale, and clinical applications, existing and potential, Int. J. Nanomedicine. 1 (2006) 297-315.

[6] Y. Matsumura, H. Maeda, A new concept for macromolecular therapeutics in cancer chemotherapy: mechanism of tumoritropic accumulation of proteins and the antitumor agent smancs, Cancer Res. 46 (1986) 6387-6392.

[7] J. Fang, H. Nakamura, H. Maeda, The EPR effect: Unique features of tumor blood vessels for drug delivery, factors involved, and limitations and augmentation of the effect, Adv. Drug Deliv. Rev. 63 (2011) 136-151.

[8] H. Hatakeyama, H. Akita, K. Kogure, M. Oishi, Y. Nagasaki, Y. Kihira, M. Ueno, H. Kobayashi, H. Kikuchi, H. Harashima, Development of a novel systemic gene delivery system for cancer therapy with a tumor-specific cleavable PEG-lipid, Gene Ther. 14 (2007) 68-77.

[9] H. Hatakeyama, H. Akita, H. Harashima, A multifunctional envelope type nano device (MEND) for gene delivery to tumours based on the EPR effect: a strategy for overcoming the PEG dilemma, Adv. Drug Deliv. Rev. 63 (2011) 152-160.

[10] T. Terada, M. Iwai, S. Kawakami, F. Yamashita, M. Hashida, Novel PEG-matrix metalloproteinase-2 cleavable peptide-lipid containing galactosylated liposomes for hepatocellular carcinoma-selective targeting, J. Control. Release. 111 (2006) 333-342.

[11] H. Xu, Y. Deng, D. Chen, W. Hong, Y. Lu, X. Dong, Esterase-catalyzed dePEGylation of $\mathrm{pH}$-sensitive vesicles modified with cleavable PEG-lipid derivatives, J. Control. Release. 130 (2008) 238-245. 
[12] G.F. Walker, C. Fella, J. Pelisek, J. Fahrmeir, S. Boeckle, M. Ogris, E. Wagner, Toward synthetic viruses: endosomal $\mathrm{pH}$-triggered deshielding of targeted polyplexes greatly enhances gene transfer in vitro and in vivo, Mol. Ther. 11 (2005) 418-425.

[13] A. Guiotto, M. Canevari, P. Orsolini, O. Lavanchy, C. Deuschel, N. Kaneda, A. Kurita, T. Matsuzaki, T. Yaegashi, S. Sawada, F.M. Veronese, Synthesis, characterization, and preliminary in vivo tests of new poly(ethylene glycol) conjugates of the antitumor agent 10-amino-7-ethylcamptothecin, J. Med. Chem. 47 (2004) 1280-1289.

[14] T. Ishida, M. Ichihara, X. Wang, K. Yamamoto, J. Kimura, E. Majima, H. Kiwada, Injection of PEGylated liposomes in rats elicits PEG-specific IgM, which is responsible for rapid elimination of a second dose of PEGylated liposomes, J. Control. Release. 112 (2006) 15-25.

[15] H. Koide, T. Asai, H. Kato, H. Ando, K. Shiraishi, M. Yokoyama, N. Oku, Size-dependent induction of accelerated blood clearance phenomenon by repeated injections of polymeric micelles, Int. J. Pharm. 432 (2012) 75-79.

[16] T. Ishida, H. Kiwada, Accelerated blood clearance (ABC) phenomenon upon repeated injection of PEGylated liposomes, Int. J. Pharm. 354 (2008) 56-62.

[17] S.K. Sahoo, T. Sawa, J. Fang, S. Tanaka, Y. Miyamoto, T. Akaike, H. Maeda, Pegylated zinc protoporphyrin: a water-soluble heme oxygenase inhibitor with tumor-targeting capacity, Bioconjug. Chem. 13 (2002) 1031-1038.

[18] J. Fang, T. Akaike, H. Maeda, Antiapoptotic role of heme oxygenase (HO) and the potential of $\mathrm{HO}$ as a target in anticancer treatment, Apoptosis. 9 (2004) 27-35.

[19] S.M. Keyse, R.M. Tyrrell, Heme oxygenase is the major 32-kDa stress protein induced in human skin fibroblasts by UVA radiation, hydrogen peroxide, and sodium arsenite, Proc. Natl. Acad. Sci. U. S. A. 86 (1989) 99-103.

[20] K. Doi, T. Akaike, S. Fujii, S. Tanaka, N. Ikebe, T. Beppu, S. Shibahara, M. Ogawa, H. Maeda, Induction of haem oxygenase-1 nitric oxide and ischaemia in experimental solid tumours and implications for tumour growth, Br. J. Cancer. 80 (1999) 1945-1954.

[21] S. Tanaka, T. Akaike, J. Fang, T. Beppu, M. Ogawa, F. Tamura, Y. Miyamoto, H. Maeda, Antiapoptotic effect of haem oxygenase-1 induced by nitric oxide in experimental solid tumour, Br. J. Cancer. 88 (2003) 902-909. 
[22] K. Mitani, H. Fujita, Y. Fukuda, A. Kappas, S. Sassa, The role of inorganic metals and metalloporphyrins in the induction of haem oxygenase and heat-shock protein 70 in human hepatoma cells, Biochem. J. 290 (Pt3) (1993) 819-825.

[23] K. Sato, K. Ito, H. Kohara, Y. Yamaguchi, K. Adachi, H. Endo, Negative regulation of catalase gene expression in hepatoma cells, Mol. Cell. Biol. 12 (1992) 2525-2533.

[24] M. Mayerhofer, K. V Gleixner, J. Mayerhofer, G. Hoermann, E. Jaeger, K.J. Aichberger, R.G. Ott, K. Greish, H. Nakamura, S. Derdak, P. Samorapoompichit, W.F. Pickl, V. Sexl, H. Esterbauer, I. Schwarzinger, C. Sillaber, H. Maeda, P. Valent, Targeting of heat shock protein 32 (Hsp32)/heme oxygenase-1 (HO-1) in leukemic cells in chronic myeloid leukemia: a novel approach to overcome resistance against imatinib, Blood. 111 (2008) 2200-2210.

[25] G. Yang, X. Nguyen, J. Ou, P. Rekulapelli, D.K. Stevenson, P.A. Dennery, Unique effects of zinc protoporphyrin on HO-1 induction and apoptosis, Blood. 97 (2001) $1306-1313$.

[26] J. Fang, T. Sawa, T. Akaike, T. Akuta, S.K. Sahoo, G. Khaled, A. Hamada, H. Maeda, In vivo antitumor activity of pegylated zinc protoporphyrin: targeted inhibition of heme oxygenase in solid tumor, Cancer Res. 63 (2003) 3567-3574.

[27] A.K. Iyer, K. Greish, T. Seki, S. Okazaki, J. Fang, K. Takeshita, H. Maeda, Polymeric micelles of zinc protoporphyrin for tumor targeted delivery based on EPR effect and singlet oxygen generation, J. Drug Target. 15 (2007) 496-506.

[28] H. Nakamura, J. Fang, B. Gahininath, K. Tsukigawa, H. Maeda, Intracellular uptake and behavior of two types zinc protoporphyrin ( $\mathrm{ZnPP}$ ) micelles, SMA-ZnPP and PEG-ZnPP as anticancer agents; unique intracellular disintegration of SMA micelles, J. Control. Release. 155 (2011) 367-375.

[29] J. Fang, K. Greish, H. Qin, L. Liao, H. Nakamura, M. Takeya, H. Maeda, HSP32 (HO-1) inhibitor, copoly(styrene-maleic acid)-zinc protoporphyrin IX, a water-soluble micelle as anticancer agent: In vitro and in vivo anticancer effect, Eur. J. Pharm. Biopharm. 81 (2012) 540-547.

[30] H. Maeda, H. Nakamura, J. Fang, The EPR effect for macromolecular drug delivery to solid tumors: Improvement of tumor uptake, lowering of systemic toxicity, and distinct tumor imaging in vivo, Adv. Drug Deliv. Rev. 65 (2013) 71-79. 
[31] F.G. Bahar, K. Ohura, T. Ogihara, T. Imai, Species difference of esterase expression and hydrolase activity in plasma, J. Pharm. Sci. 101 (2012) 3979-3988.

[32] [34] S. Rakash, Role of proteases in cancer: A review, Biotechnol. Mol. Biol. Rev. 7 (2012) 90-101.

[33] J.M. Jessup, Cathepsin B and other proteases in human colorectal carcinoma, Am. J. Pathol. 145 (1994) 253-62.

[34] T. Aboul-Fadl, M. Sheha, A. El-Azab, H. Abdel-Aziz, Amantadine Amides Prodrugs as Hepatic Delivery Systems to Enhance its Activity against HCV, Dig. J. Nanomater. Biostructures. 6 (2011) 1675-1683.

[35] W. Wichitnithad, U. Nimmannit, P.S. Callery, P. Rojsitthisak, Effects of different carboxylic ester spacers on chemical stability, release characteristics, and anticancer activity of mono-PEGylated curcumin conjugates, J. Pharm. Sci. 100 (2011) 5206-5218. 


\section{Figure Legend}

Figure 1. Chemical structures of (A) esPEG-ZnPP, (B) etPEG-ZnPP, (C) free ZnPP, (D) ZnPPED, (E) mono-succinyl ZnPPED, and (F) bis-succinyl ZnPPED.

Figure. 2. Analyses of PEG-ZnPP by HPLC after treatment with blood plasma or with supernatant of tumor homogenates. (A) The peak of intact PEG-ZnPP, the original sample (A-i), and peaks of the cleavage products after incubation of esPEG-ZnPP with $10 \%$ mouse blood plasma for $6 \mathrm{~h}$ (A-ii). Similar results were obtained for cleavage products of esPEG-ZnPP incubated with supernatant of mouse and human tumor homogenates. (B) Peaks of standards: free ZnPP, ZnPPED, and succinyl ZnPPEDs (mono- and bis-succinyl ZnPPED, synthesized), all at about $10 \mathrm{ng} / 20 \mu \mathrm{L}$, measured by using HPLC. Note that Figure A-i and A-ii are plotted at same y-axis, but Figure B is at different scale.

Figure 3. Stability of es- and et- PEG-ZnPP in $10 \%$ blood plasma from different species and effect of DFP (1 mM) added to $10 \%$ human or mouse blood plasma at $\mathrm{pH} 7.4,37{ }^{\circ} \mathrm{C}$, analyzed by using HPLC. (A, B) Changes in peak 1 for esPEG-ZnPP and etPEG-ZnPP (A), changes in peak 2 for esPEG-ZnPP (B), after incubation in 10\% blood plasma of human, rabbit, mouse, or rat blood plasma. (C, D) Changes in peak $1(\mathrm{C})$, peak 2 (D) for esPEG-ZnPP after incubation in $10 \%$ human or mouse blood plasma with (solid) or without (open) $1 \mathrm{mM}$ DFP. Symbols in (B) and (D) are same as those in (A) and (C), respectively. Values are means \pm S.E. $(n=3)$. H, Human; Rab, Rabbit; M, Mouse; R, Rat.

Figure 4. Cleavability of PEG chains from esPEG-ZnPP and etPEG-ZnPP after incubation with supernatant of mouse S-180 tumor homogenates (final conc.: 2 or $5 \mathrm{mg} / \mathrm{mL}$ protein) at pH 6.2, $37{ }^{\circ} \mathrm{C}$, analyzed by HPLC. Yield of peak 3 (A), peak 4 (B), and peak 5 (C) after incubation of esPEG-ZnPP or etPEG-ZnPP with supernatant of tumor homogenate. Notations of bars in (A) apply to (B) and (C) as well. Values are means \pm S.E. $(n=3)$.

Figure 5. Effect of protease inhibitors on depegylation from esPEG-ZnPP incubated with supernatant of mouse S-180 tumor homogenates (final conc.: $5 \mathrm{mg} / \mathrm{mL}$ protein) at $\mathrm{pH} 6.2$, $37^{\circ} \mathrm{C}$, analyzed by HPLC. Yield of peak 3 (A), peak 4 (B), and peak 5 (C) after incubation of esPEG-ZnPP with supernatant of tumor homogenate in the presence or absence of the 
indicated protease inhibitors. Notations of bars in (A) apply to (B) and (C) as well. Values are means \pm S.E. $(n=3) .{ }^{*} p<0.05$, a significant difference from tumor homogenate $(\mathrm{T})$, according to Student's $t$ test.

Figure 6. Cleavability of the PEG chains from esPEG-ZnPP after incubation with supernatants of homogenates of different mouse tissues, analyzed by HPLC. Yield of cleavage products (total of peaks 3-5 combined) after incubation of esPEG-ZnPP with supernatants of tissue homogenates of mouse spleen, kidney, and liver (final conc.: $2 \mathrm{mg} / \mathrm{mL}$ protein) at $\mathrm{pH}$ 7.4 or with supernatant of mouse tumor homogenates at $\mathrm{pH} 6.2,37^{\circ} \mathrm{C}$. Values are means \pm S.E. $(n=3) .{ }^{*} p<0.05$, a significant difference from no supernatant, according to Student's $t$ test.

Figure 7. Cleavability of PEG chains from esPEG-ZnPP after incubation with supernatants of human colorectal tumor homogenates at $\mathrm{pH} 6.2$ or human normal large intestine homogenates (final conc.: $5 \mathrm{mg} / \mathrm{mL}$ protein) at $\mathrm{pH} 7.4,37^{\circ} \mathrm{C}$, analyzed by HPLC. (A) Yield of cleavage products (total of peaks 3-5 combined) after incubation of esPEG-ZnPP with supernatants of tumor homogenates. (B) Yield of cleavage products (total of peaks 3-5 combined) similar to (A) in the presence or absence of activator of thiol proteases, $N$-acetylcysteine. (C) Similar to (A), tumor tissue vs normal large intestine at $24 \mathrm{~h}$ after incubation. Values are means \pm S.E. ( $n$ $=3-5) .{ }^{*} p<0.05$, a significant difference according to Student's $t$ test.

Figure 8. Intracellular uptake of esPEG-ZnPP, etPEG-ZnPP, free ZnPP, and ZnPPED. (A, B) Intracellular uptake in K562 cells (A) measured by FACS analyses and in HT-29 cells (B) quantified by fluorescence intensity. The insets show comparison of intracellular uptake of esand et- PEG-ZnPP after $8 \mathrm{~h}$ in K562 cells (A), after $16 \mathrm{~h}$ and $24 \mathrm{~h}$ in HT-29 cells (B). Values are means \pm S.E. $(n=3) .{ }^{*} p<0.05$, a significant difference according to Student's $t$ test. (C) Confocal fluorescence microscopic analysis of KYSE150 cells treated with esPEG-ZnPP, etPEG-ZnPP, free ZnPP, or ZnPPED after $2.5 \mathrm{~h}$ incubation.

Figure 9. Cytotoxicity of esPEG-ZnPP, etPEG-ZnPP, free ZnPP, and ZnPPED, and in vivo antitumor effect of esPEG-ZnPP and etPEG-ZnPP. (A, B) Cytotoxicity of esPEG-ZnPP, etPEG-ZnPP, free ZnPP, and ZnPPED to K562 cells (A) and HT-29 cells (B) after $72 \mathrm{~h}$ 
treatment, analyzed by MTT assay. Values are means \pm S.E. (C) Antitumor effect of esPEG-ZnPP and etPEG-ZnPP at $15 \mathrm{mg} / \mathrm{kg}$ of ZnPP equivalent in S-180 tumor bearing male ddY mice. Vertical arrows indicate injection time of drugs. Values are means \pm S.E. $(n=5)$. $*_{p} p 0.05$, a significant difference from etPEG-ZnPP, according to Student's $t$ test.

Figure 10. Tissue distribution and PEG cleavability of esPEG-ZnPP and etPEG-ZnPP in S-180 tumor bearing male ddY mice after administration at $40 \mathrm{mg} / \mathrm{kg}$ of $\mathrm{ZnPP}$ equivalent, measured by HPLC. (A) Tissue distribution (total of peaks 1-5 combined) of esPEG-ZnPP and etPEG-ZnPP at $24 \mathrm{~h}$ after administration. The inset shows amount of each PEG-ZnPP in tumor tissue with magnified scale. $(\mathrm{B}, \mathrm{C})$ The amount of each peak in each tissue at $24 \mathrm{~h}$ after administration of esPEG-ZnPP (B) and etPEG-ZnPP (C), using results in (A). The insets show amount of each peak in tumor tissue with magnified scale, respectively. Values are means \pm S.E. $(n=3)$. Amount of peak is described as the area of the peak. 
Table $1 . \mathrm{IC}_{50}, \mathrm{IC}_{30}$, and $\mathrm{IC}_{10}$ of esPEG-ZnPP, etPEG-ZnPP, free ZnPP, and ZnPPED against K562 cells.

\begin{tabular}{cccc}
\hline Drugs & $\mathrm{IC}_{50}(\mu \mathrm{M})$ & $\mathrm{IC}_{30}(\mu \mathrm{M})$ & $\mathrm{IC}_{10}(\mu \mathrm{M})$ \\
\hline esPEG-ZnPP & 2.88 & 1.76 & 0.61 \\
etPEG-ZnPP & 39.1 & 2.71 & 0.41 \\
Free ZnPP & 0.97 & 0.65 & 0.32 \\
ZnPPED & 0.85 & 0.56 & 0.24 \\
\hline
\end{tabular}

Data are calculated from the results in Figure 8D

Table 2. $\mathrm{IC}_{50}, \mathrm{IC}_{30}$, and $\mathrm{IC}_{10}$ of esPEG-ZnPP, etPEG-ZnPP, free $\mathrm{ZnPP}$, and $\mathrm{ZnPPED}$ against HT-29 cells.

\begin{tabular}{cccc}
\hline Drugs & $\mathrm{IC}_{50}(\mu \mathrm{M})$ & $\mathrm{IC}_{30}(\mu \mathrm{M})$ & $\mathrm{IC}_{10}(\mu \mathrm{M})$ \\
\hline esPEG-ZnPP & 27.7 & 2.77 & 1.03 \\
etPEG-ZnPP & $>100$ & 73.4 & 2.61 \\
Free ZnPP & 4.16 & 1.64 & 0.14 \\
ZnPPED & 2.82 & 0.65 & 0.15 \\
\hline
\end{tabular}

Data are calculated from the results in Figure 8E 
Figure 1
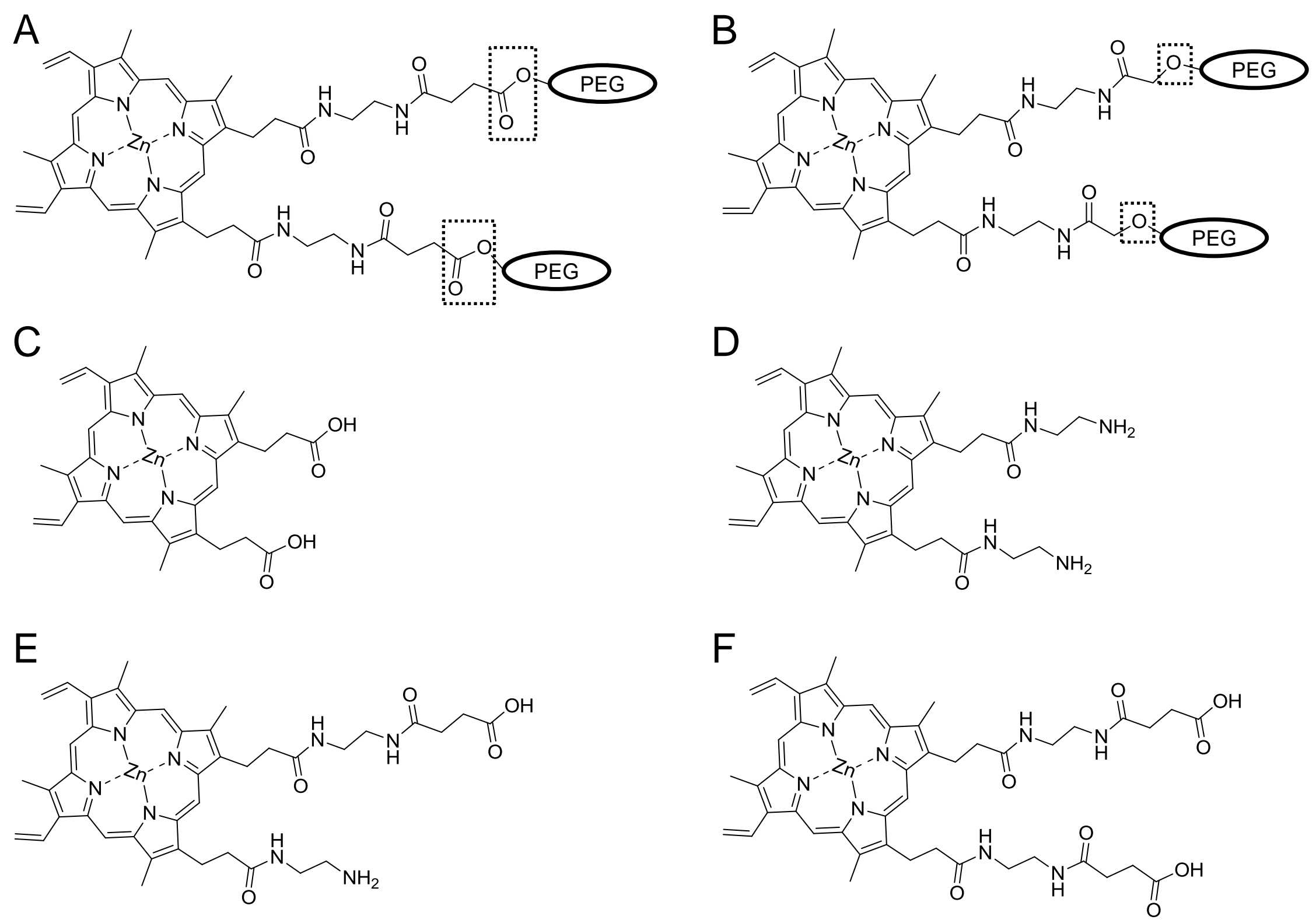


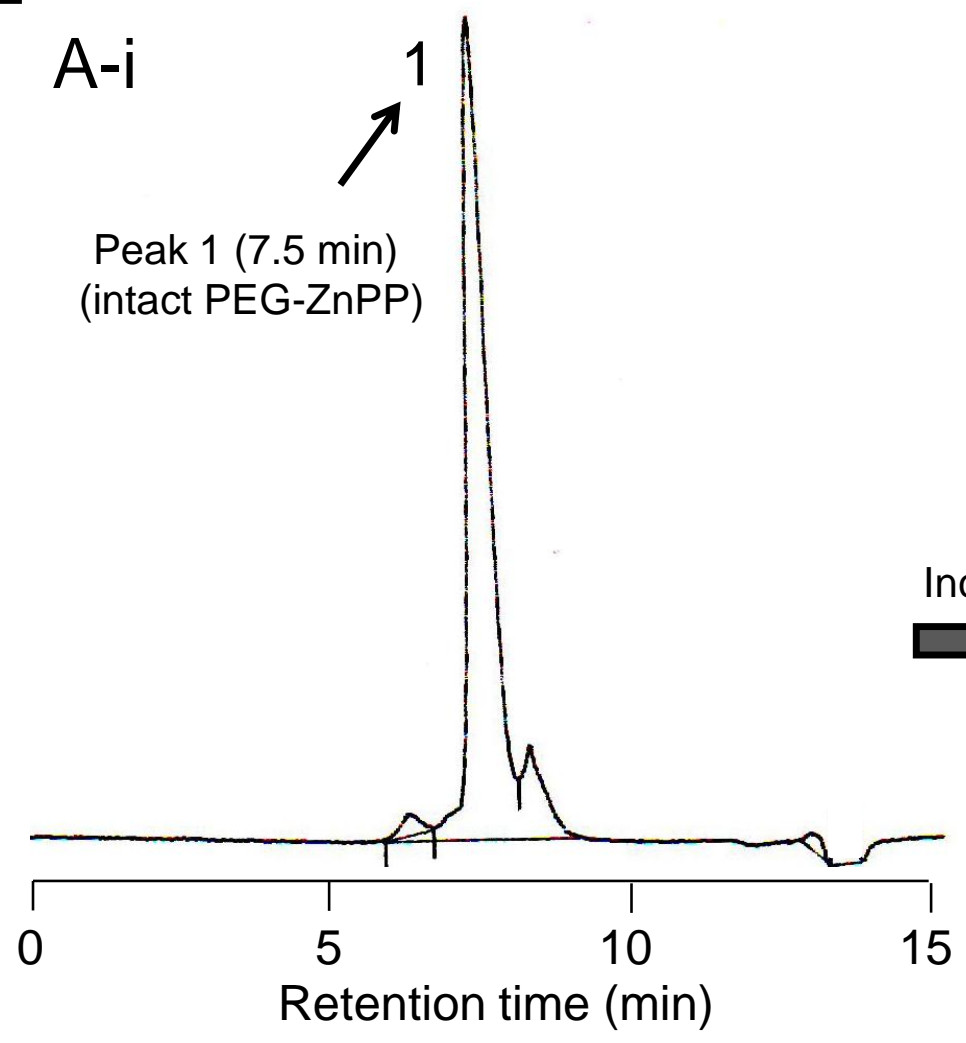

A-ii
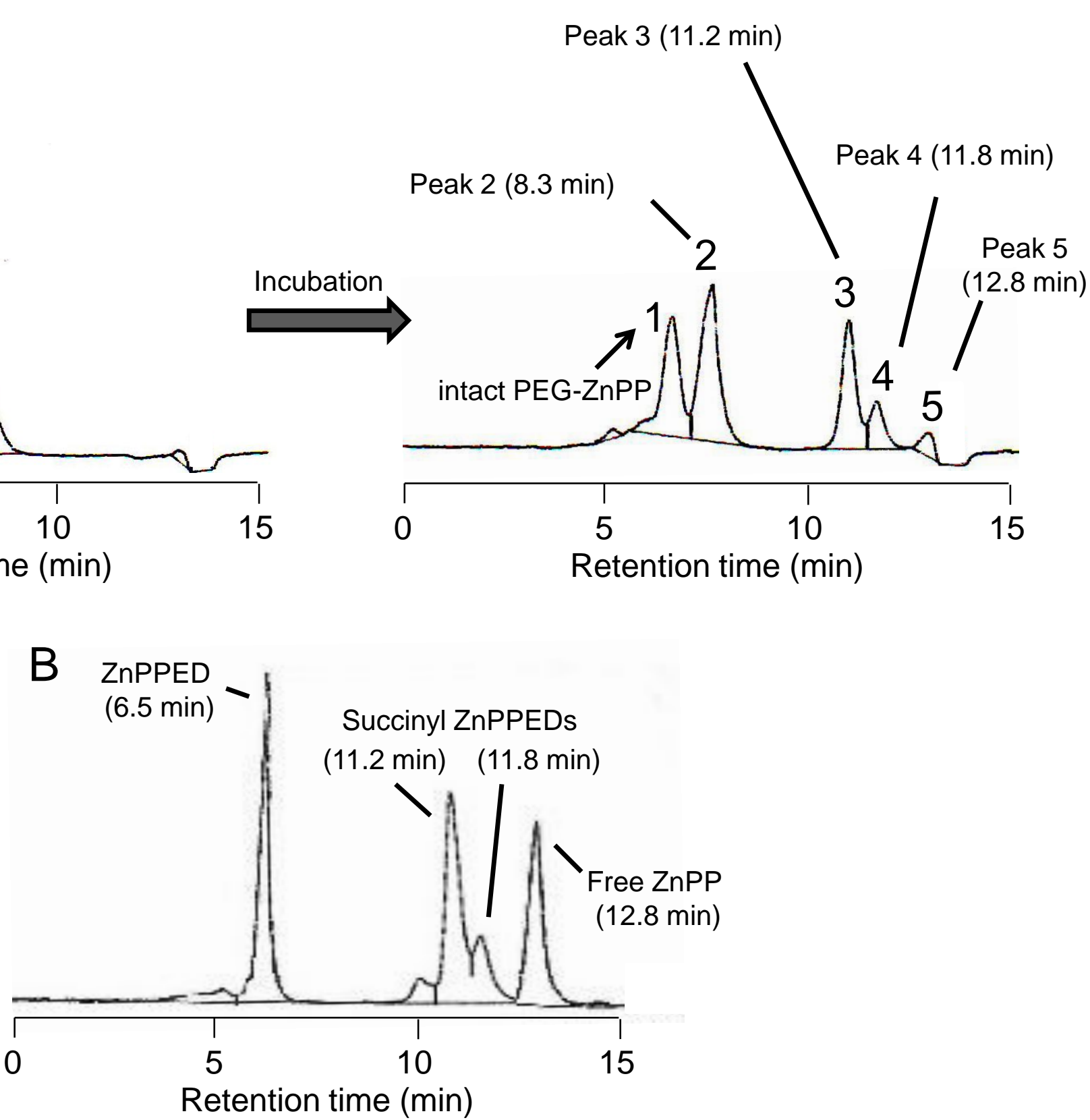
Figure 3
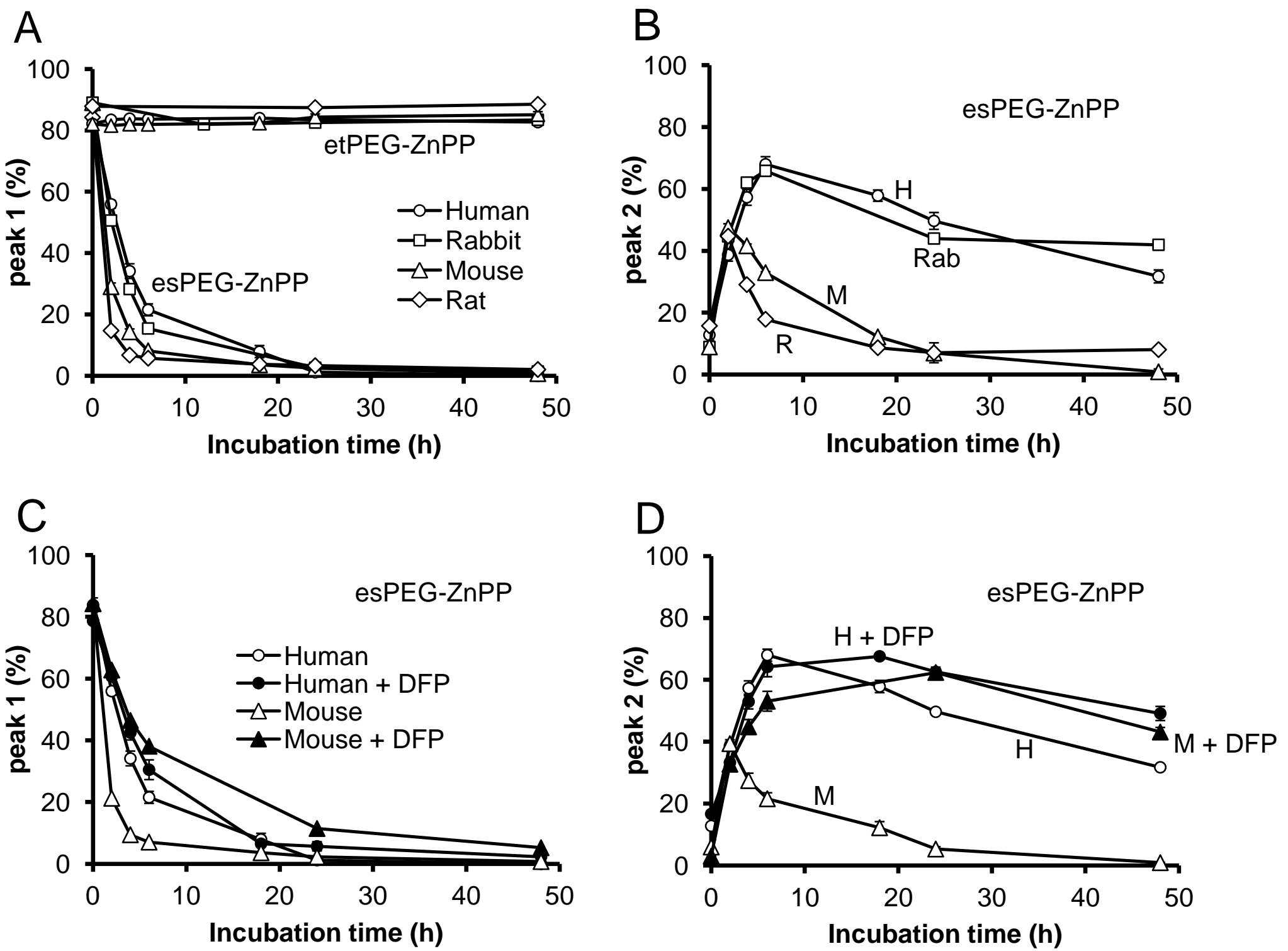
A 30, (1) DesPEG-ZnPP (no supernatant)
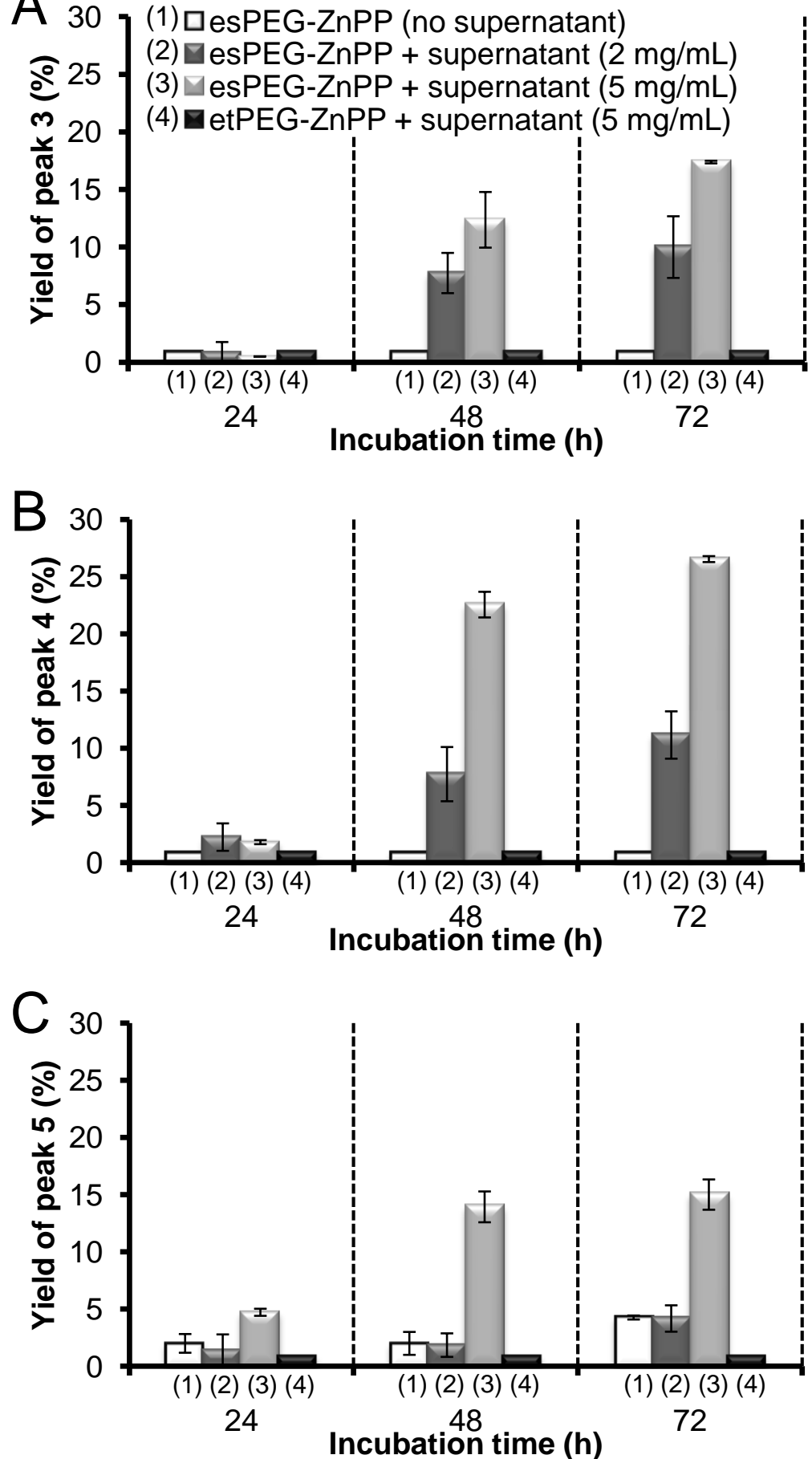
A $30,(1) \square$ Supernatant of mouse $S-180$ tumor homogenate (T)
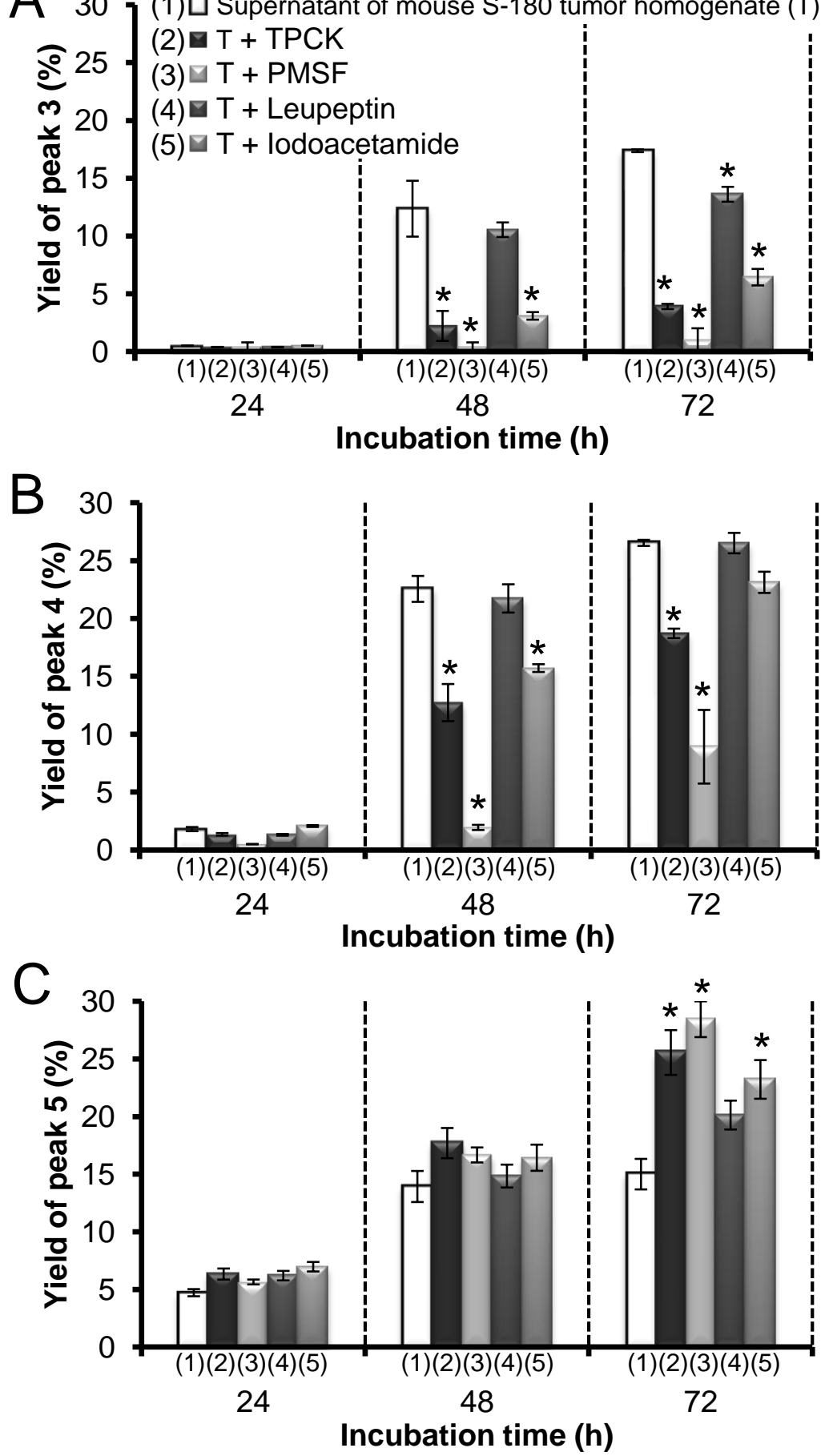


\section{Figure 6}

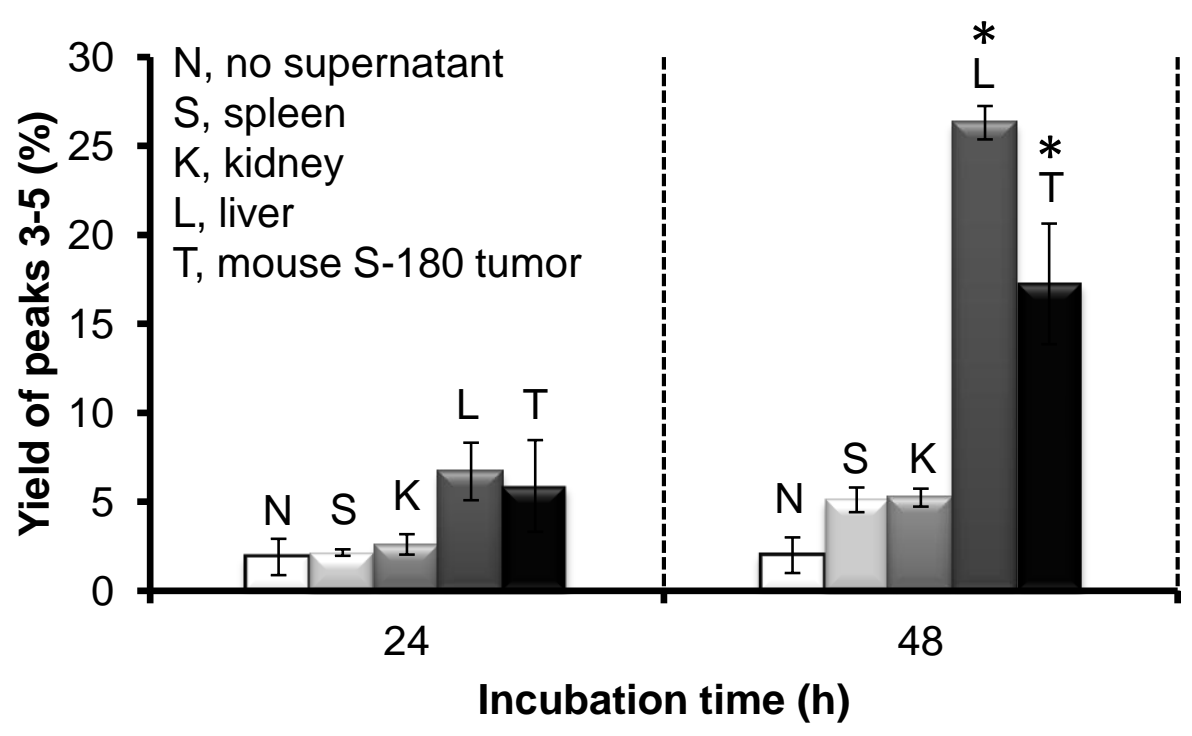


A

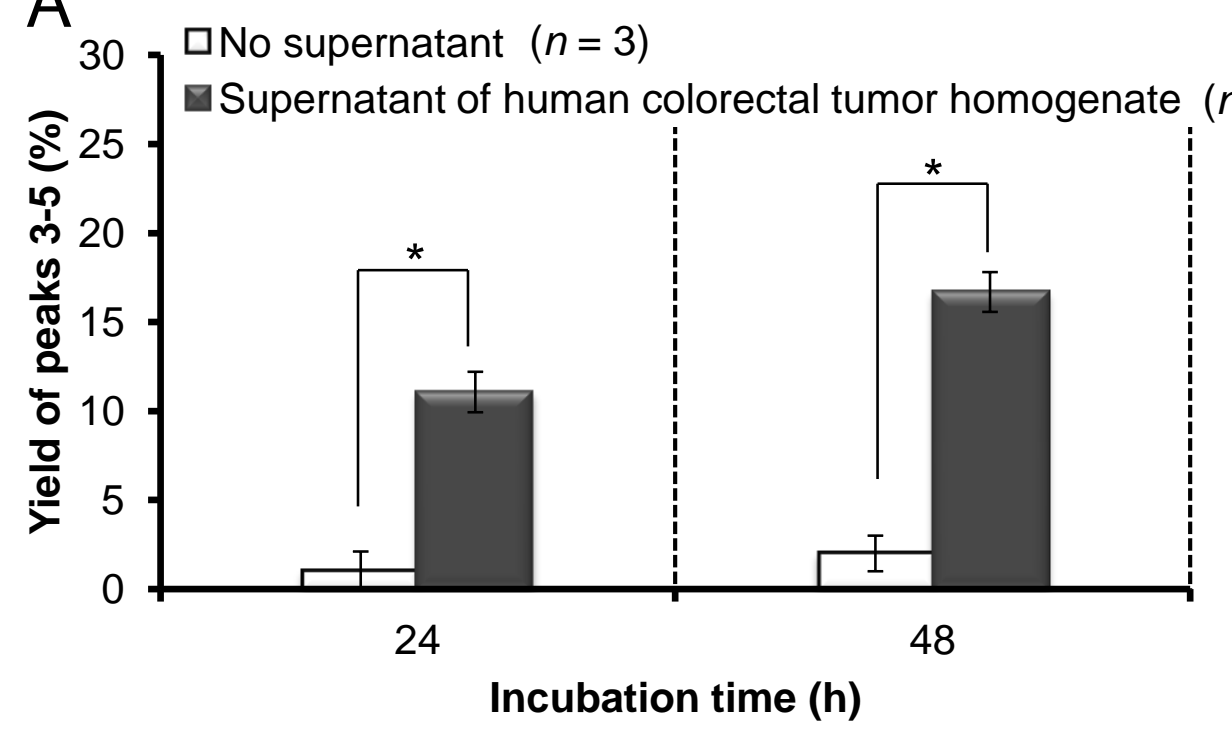

B
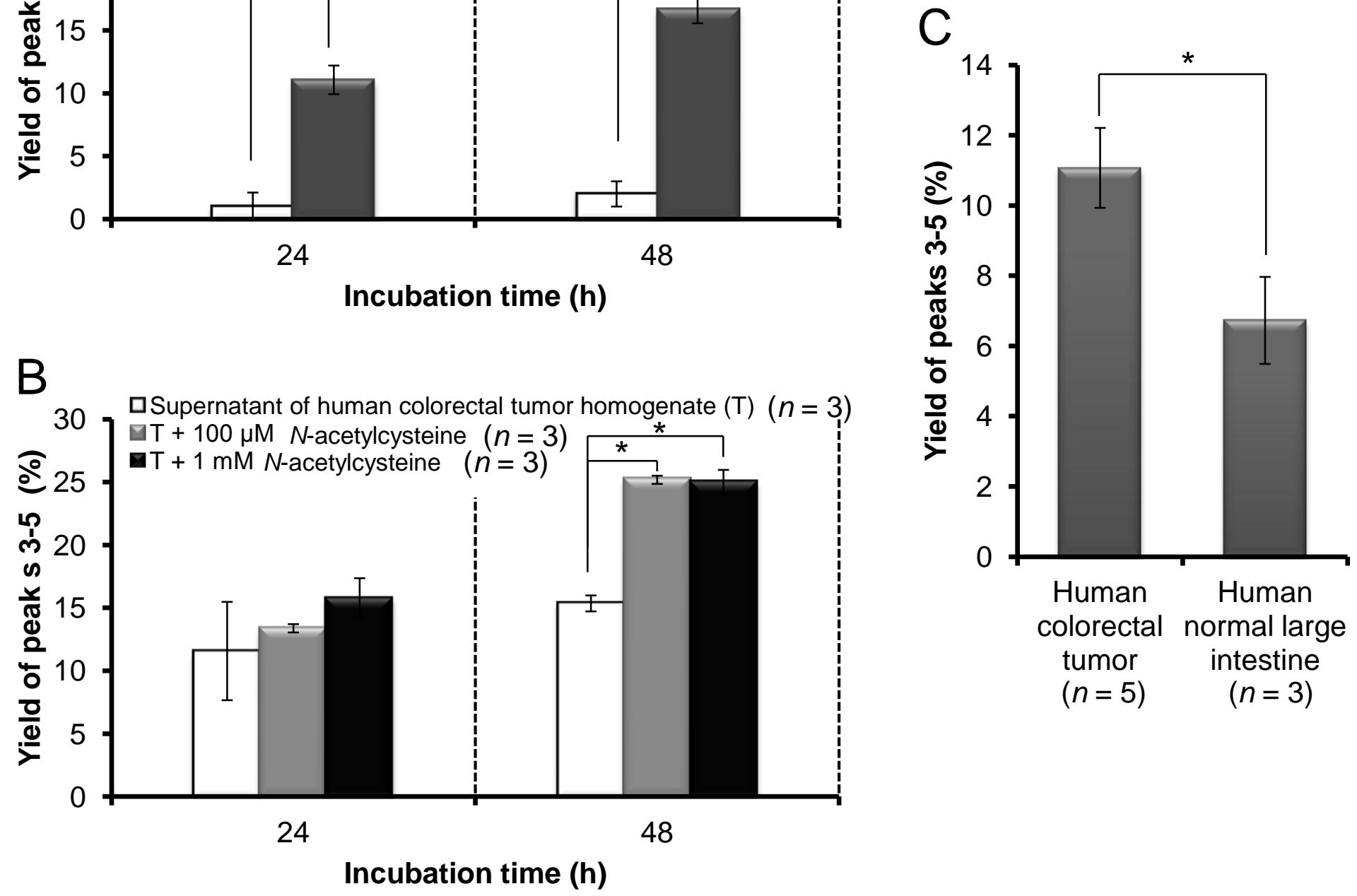
A
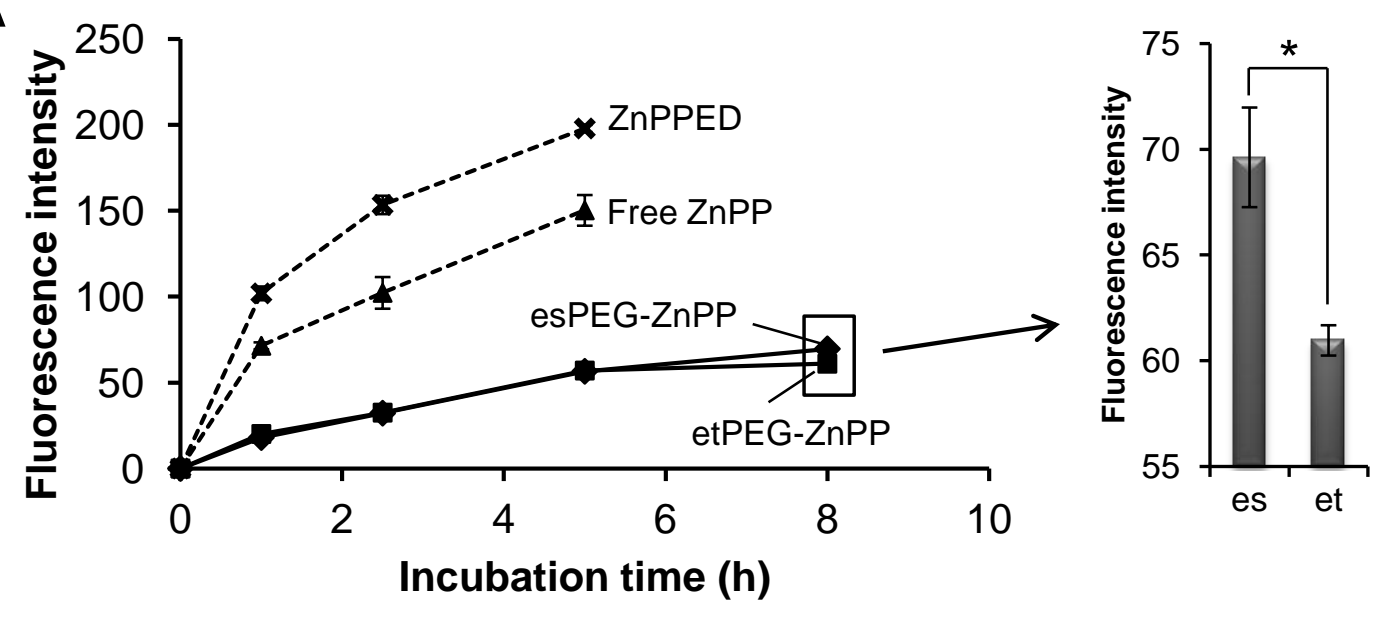

B
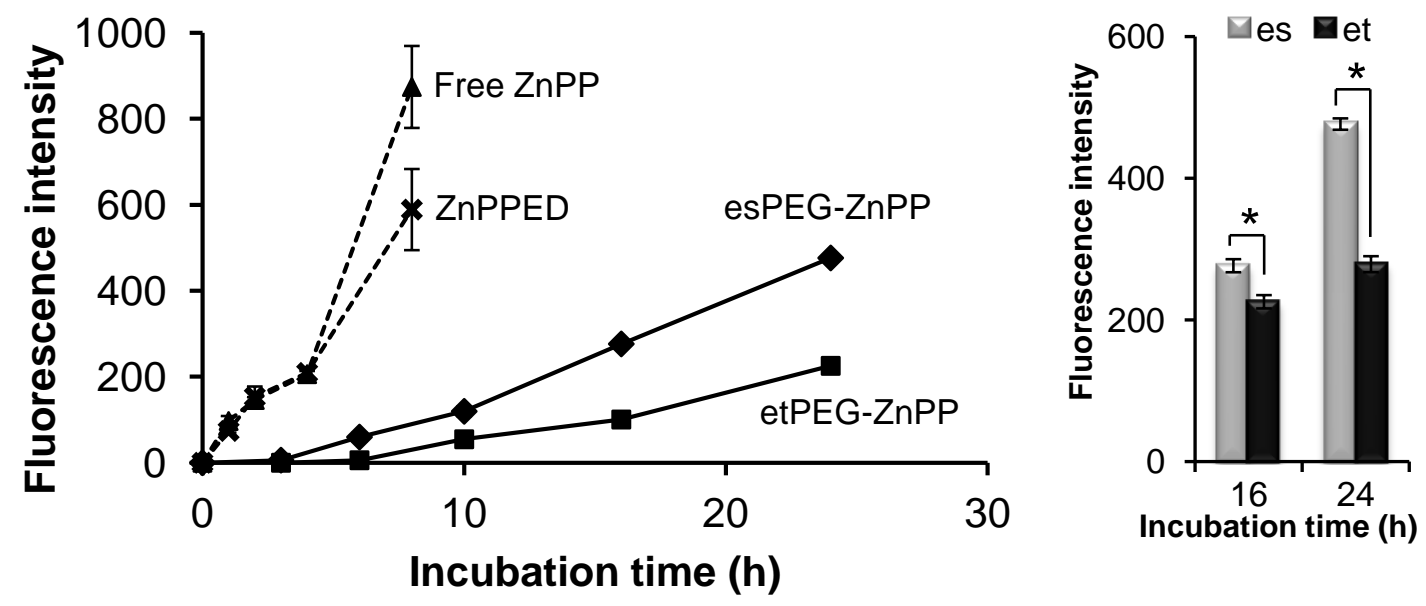

C

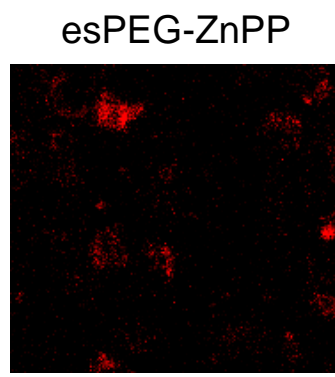

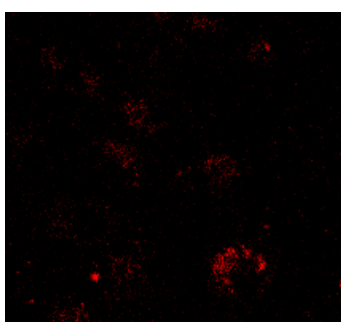

Free ZnPP

ZnPPED

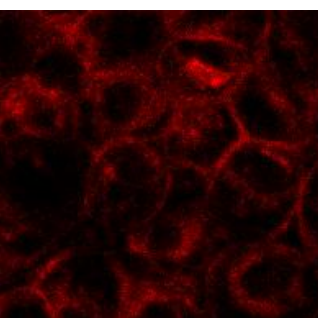

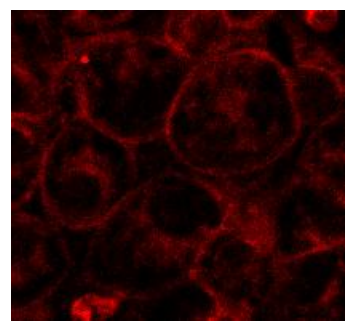



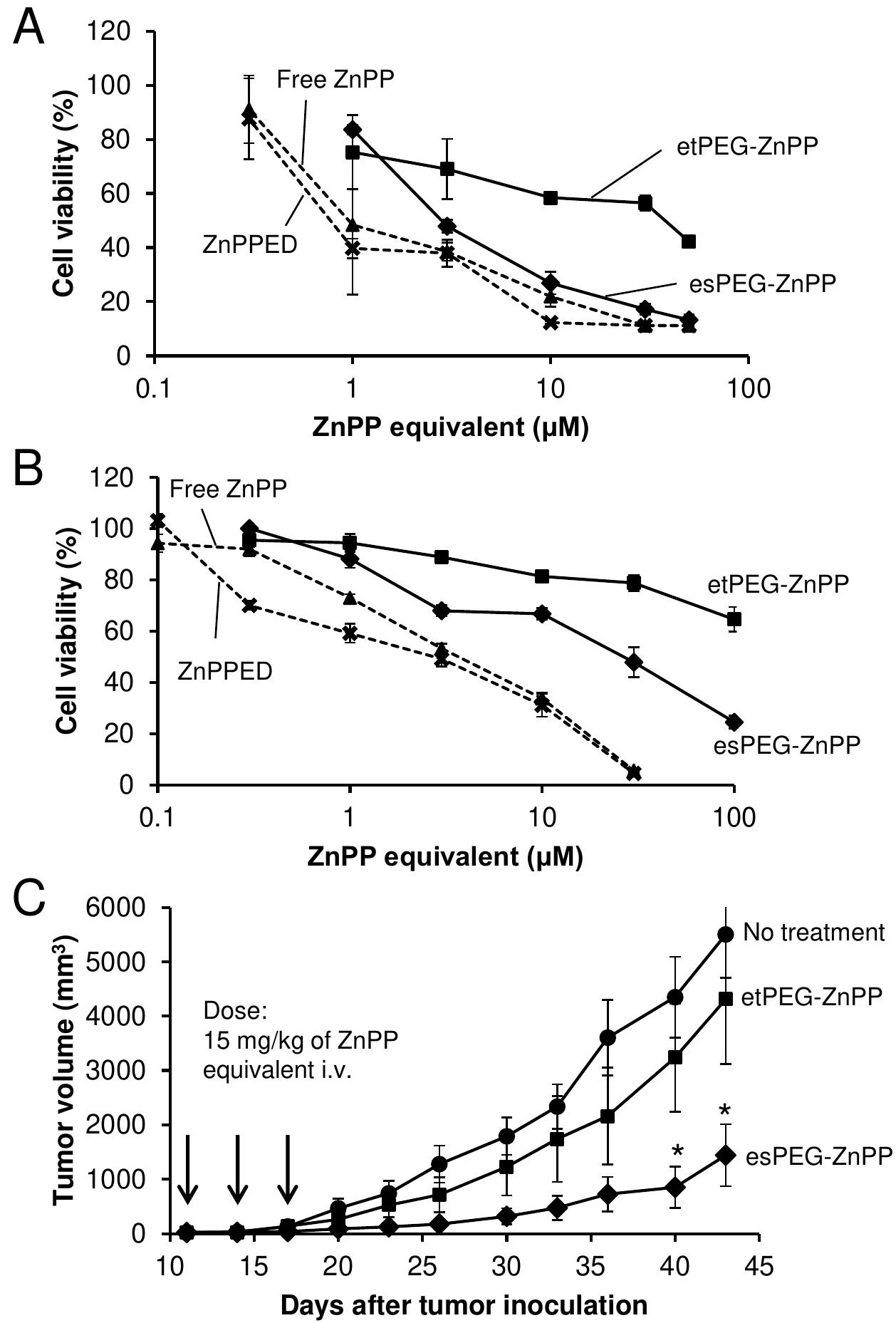

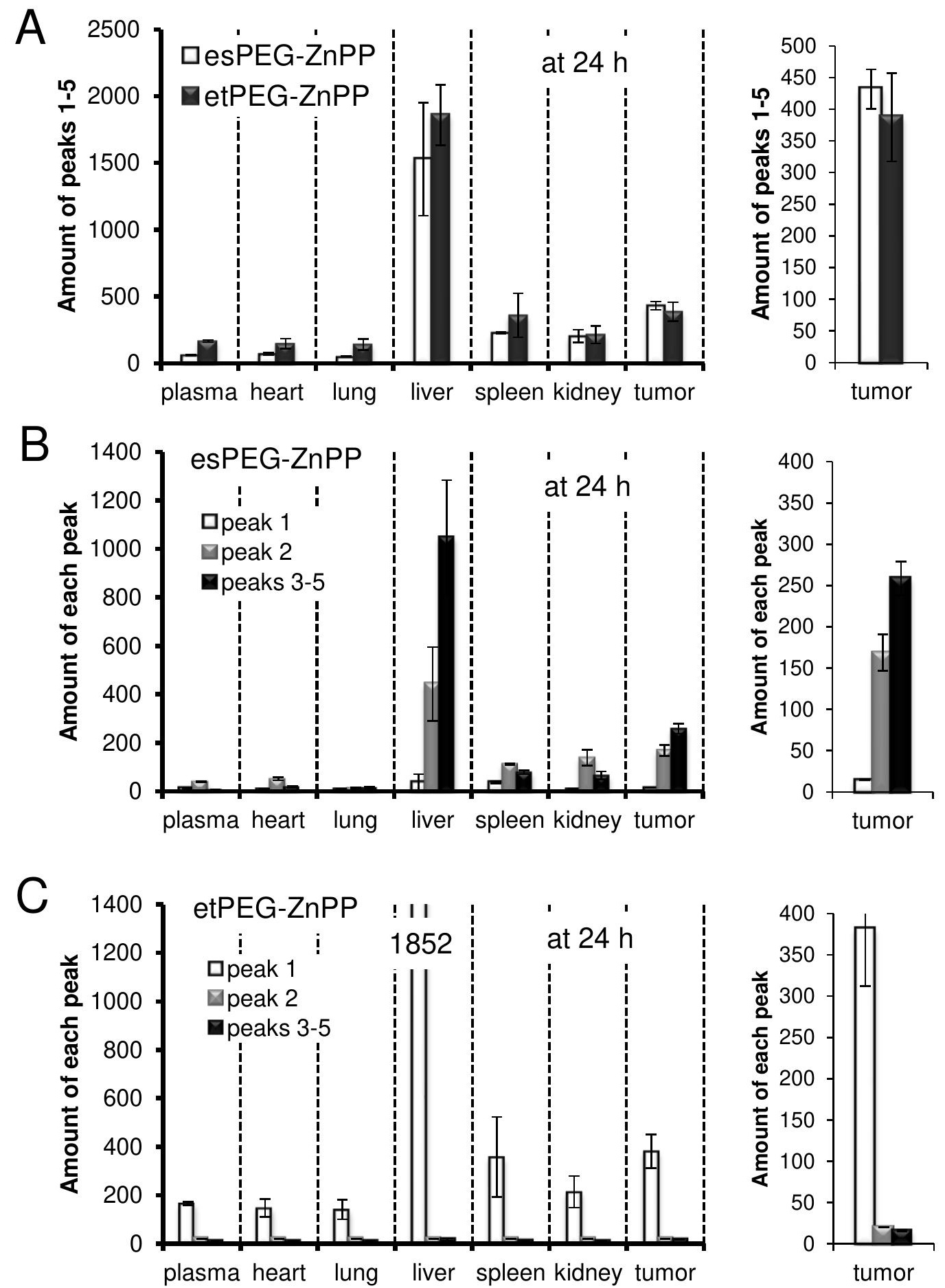


\section{Graphical Abstract ${ }_{\text {Ester bonded PEG-ZnPP (esPEG-ZnPP) }}$}

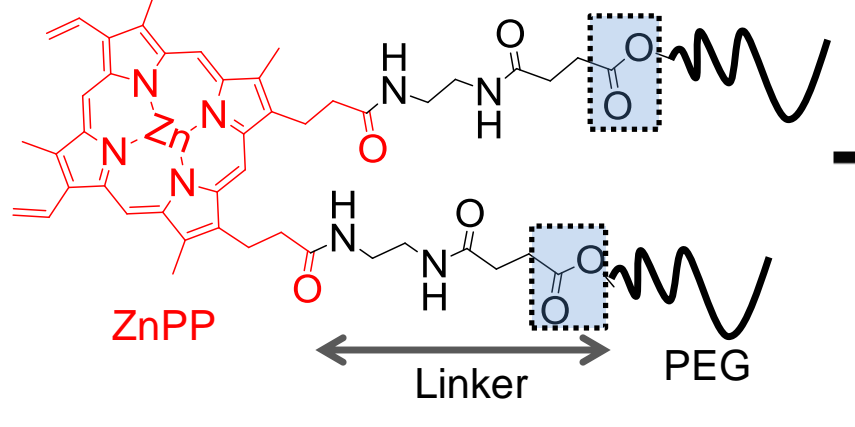

Micelle of esPEG-ZnPP
Ether bonded PEG-ZnPP (etPEG-ZnPP)

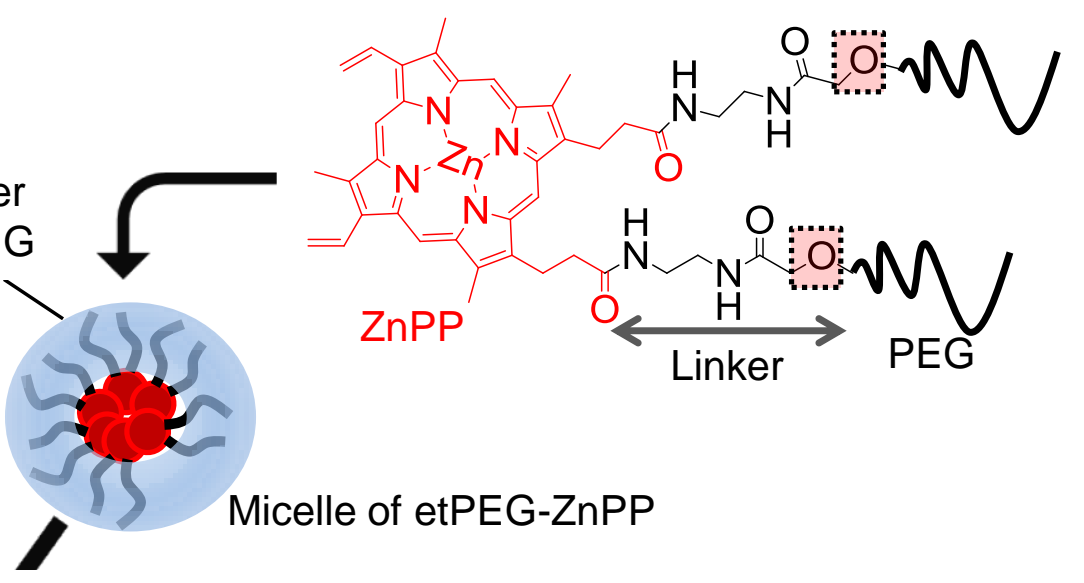

Tumor accumulation
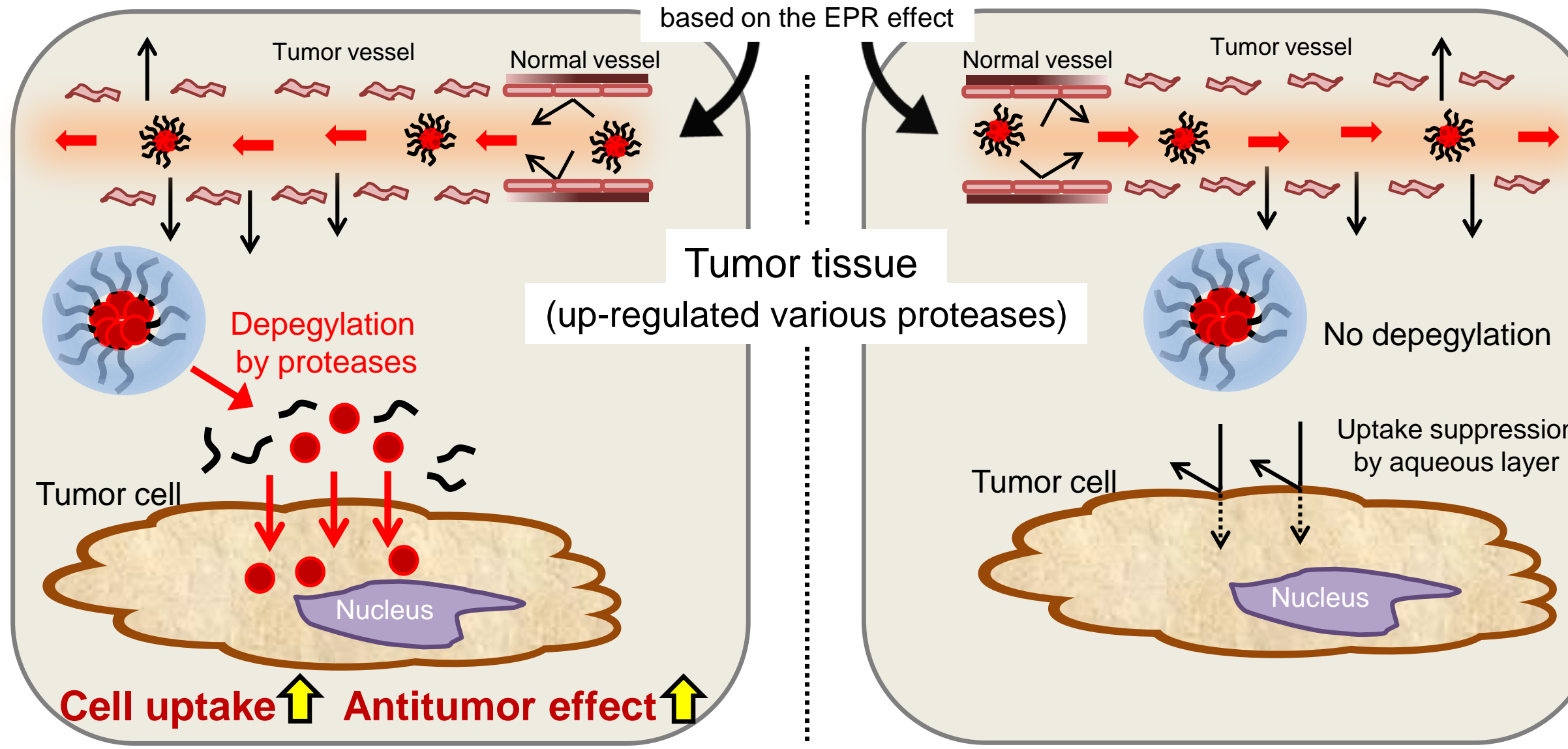

\section{various proteases)}

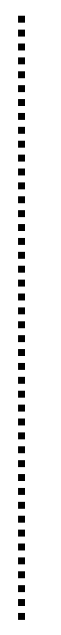

No depegylation

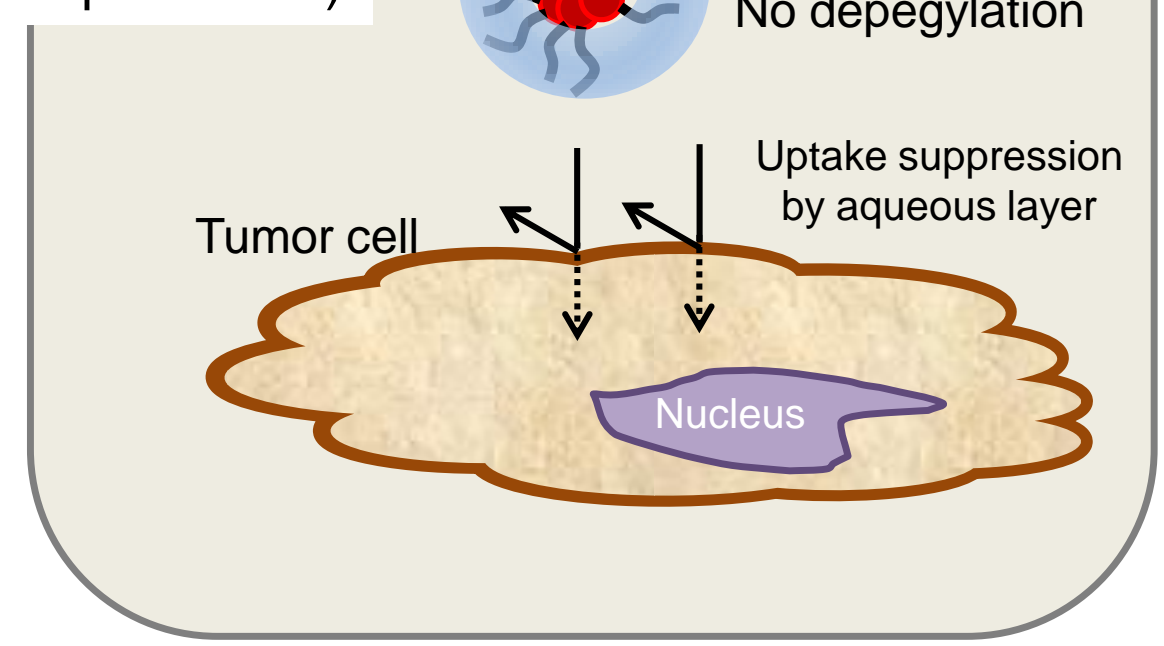

